\title{
Mesh generation for aerospace applications
}

\author{
N P WEATHERILL \\ Institute for Numerical Methods in Engineering, University College of \\ Swansea, Swansea SA2 8PP, UK \\ MS received 18 January 1991
}

\begin{abstract}
In recent years there has been much research activity in the field of compressible flow simulation for aerodynamic applications. In the 1970's and 1980's the advances in the numerical solution of the Full Potential and Euler equations made, in principle, the inviscid flow simulation around complex aerodynamic shapes possible. At this stage much attention was focused on methods capable of generating meshes on which such calculations could be performed.

In this paper an overview is presented of some techniques which have been developed to generate meshes for aerospace applications. Structured mesh generation techniques are discussed and their application to complicated shapes utilising the multiblock approach is highlighted. Unstructured mesh generation methods are also discussed with particular emphasis given to the Delaunay triangulation method. Finally, the advantages and disadvantages of the structured and unstructured approaches are discussed and new work is presented which attempts to utilise both these approaches in an efficient and flexible manner.
\end{abstract}

Keywords. Structured mesh generation; unstructured mesh generation; inviscid flow simulation; meshes for aerospace applications; Delaunay triangulation method; computation fluid dynamics.

\section{Structured meshes from partial differential equations}

\subsection{Introduction}

The majority of problems in physics and engineering can be described in terms of partial differential equations (Sneddon 1957). Moreover, many of these problems fall naturally into one of three physical categories: equilibrium problems, eigenvalue problems and propagation problems. At first it may appear inappropriate to suggest that before solving such problems by numerical methods a system of partial differential equations should be solved to determine the mesh! Not only is this approach viable but the generation of meshes from the solution of partial differential equations is today a popular approach. The properties of meshes generated by this approach are intimately connected to the properties of the partial differential equations used as the mesh generation equations. 


\subsection{Elliptic systems}

1.2a Laplace and Poisson equations: The motivation for the use of elliptic equations as generators of mesh points can be derived from a number of sources. The nature of elliptic equations is to smooth boundary data and this affords a most desirable property. In fact, many elliptic equations are based around Laplace's equation which is well known as a filter or smoothing operator. In two dimensions, Laplace's equation with Cauchy-Riemann type boundary conditions can be used to generate conformal mappings (see $\$ 3$ ). In fact, the real and imaginary parts of an analytic transformation are harmonic functions. An alternative viewpoint, and one which is most appropriate in computational fluid dynamics, is to note that a two-dimensional, inviscid, steady, incompressible flow is described in terms of Laplace's equation in the potential function $\varphi$ or the stream function $\psi$, i.e.

or

$$
\partial^{2} \varphi / \partial x^{2}+\partial^{2} \varphi / \partial y^{2}=\varphi_{x x}+\varphi_{y y}=0
$$

$$
\partial^{2} \psi / \partial x^{2}+\partial^{2} \psi / \partial y^{2}=\psi_{x x}+\psi_{y y}=0
$$

Given appropriate boundary conditions for (1) the solution represents the streamlines and potential lines of the flowfield.

To utilise these ideas for mesh generation, it proves more convenient to transform these equations so that $x$ and $y$ become the dependent variables. In such a case it is then possible to apply boundary conditions to $x$ and $y$ which, in general, will be the known boundary coordinate values of the geometrical domain. If (1) are generalised to include source functions $P$ and $Q$ and, for notational convenience, $\xi$ and $\eta$ replace $\varphi$ and $\psi$, respectively, then (1) can be written as

where

$$
\begin{aligned}
& \xi_{x x}+\xi_{y y}=P(\xi, \eta), \\
& \eta_{x x}+\eta_{y y}=Q(\xi, \eta),
\end{aligned}
$$

and

$$
\xi=\xi(x, y)
$$

$$
\eta=\eta(x, y)
$$

Hence, using these relations leads to the system of coupled nonlinear equations in $x$ and $y$, namely,

with

$$
\begin{aligned}
& \alpha x_{\xi \xi}-2 \beta x_{\xi \eta}+\gamma x_{\eta \eta}=-J^{2}\left(x_{\xi} P+x_{\eta} Q\right), \\
& \alpha y_{\xi \xi}-2 \beta y_{\xi \eta}+\gamma y_{\eta \eta}=-J^{2}\left(y_{\xi} P+y_{\eta} Q\right),
\end{aligned}
$$

$$
\alpha\left(x_{\eta}^{2}+y_{\eta}^{2}\right), \beta=\left(x_{\xi} x_{\eta}+y_{\xi} y_{\eta}\right), \gamma=\left(x_{\xi}^{2}+y_{\xi}^{2}\right) \text {. }
$$

Equations (3) with (4), which were derived and popularised by Thompson et al (1974), are the transformed equations and form the system of mesh generation equations subject to applied boundary conditions. It is noted that $\alpha, \beta$ and $\gamma$ are the metric coefficients and if $\beta=0$ everywhere the mesh is orthogonal. There is a temptation to substitute $\beta=0$ in (3). However, this does not impose the orthogonality condition, and in fact, can lead to cross-over within the mesh for some boundary shapes.

The solution of this system of equations can be obtained by using an appropriate linearisation and central difference representation for the derivatives. For $P=Q=0$, 
the residual on a square mesh with $\xi=i h,(i=0,1,2, \ldots, m-1, m), \eta=j h,(j=0,1,2, \ldots$, $n-1, n)$ can be represented as

with

$$
\begin{array}{r}
R_{i, j}^{n}=\alpha_{i, j}\left(u_{i+1, j}^{n}-2 u_{i, j}^{n}+u_{i-1, j}^{n}\right)-2 \beta_{i, j}\left(u_{i+1, j+1}^{n}-u_{i-1, j+1}^{n}-\right. \\
\left.-u_{i+1, j-1}^{n}+u_{i-1, j-1}^{n}\right)+\gamma_{i, j}\left(u_{i, j+1}^{n}-2 u_{i, j}^{n}+u_{i, j-1}^{n}\right)
\end{array}
$$

$$
\begin{aligned}
& \alpha_{i, j}=\left(x_{i, j+1}^{n}-x_{i, j-1}^{n}\right)^{2}+\left(y_{i, j+1}^{n}-y_{i, j-1}^{n}\right)^{2}, \\
& \beta_{i, j}=\left(x_{i+1, j}^{n}-x_{i-1, j}^{n}\right)\left(x_{i, j+1}^{n}-x_{i, j-1}^{n}\right)+\left(y_{i+1, j}^{n}-y_{i-1, j}^{n}\right)\left(y_{i, j+1}^{n}-y_{i, j-1}^{n}\right), \\
& \gamma_{i, j}=\left(x_{i+1, j}^{n}-x_{i-1, j}^{n}\right)^{2}+\left(y_{i+1, j}^{n}-y_{i-1, j}^{n}\right)^{2},
\end{aligned}
$$

and where $u=(x, y)$ and $u_{i, j}^{n}$ represents the unknown at the point $(i, j)$ at iteration level $n$. It follows that the solution to (3) can be obtained using the stationary and linear successive point over-relaxation scheme,

$$
u_{i, j}^{n+1}=u_{i, j}^{n}+\omega R_{i, j}^{n} / 2(\alpha+\gamma)
$$

where $\omega$ is the relaxation parameter, defined in the interval [1,2]. A similar procedure is adopted to include the source functions. Other solution routines can be used including successive line over-relaxation, approximate factorisation and alternating direction implicit schemes. The convergence can be accelerated by using multiple grids (Forsey \& Billing 1988; Thames 1984).

The ideas expressed here extend into three dimensions. The system of Poisson equations is

$$
\begin{aligned}
& \xi_{x x}+\xi_{y y}+\xi_{z z}=P(\xi, \eta, \zeta), \\
& \eta_{x x}+\eta_{y y}+\eta_{z z}=Q(\xi, \eta, \zeta), \\
& \zeta_{x x}+\zeta_{y y}+\zeta_{z z}=R(\xi, \eta, \zeta),
\end{aligned}
$$

in which $P, Q$, and $R$ are now the source terms. Transforming the variables in (8) leads to

where

$$
\begin{aligned}
& \alpha_{11} r_{\xi \xi}+\alpha_{22} r_{\eta \eta}+\alpha_{33} r_{\zeta \zeta}+2\left(\alpha_{12} r_{\xi \eta}+\alpha_{13} r_{\xi \zeta}+\alpha_{23} r_{\eta \zeta}\right) \\
& \quad=-J^{2}\left(P r_{\xi}+Q r_{\eta}+R r_{\zeta}\right),
\end{aligned}
$$

$$
r=(x, y, z)^{T}, \alpha_{i j}=\sum \gamma_{m i} \gamma_{m j},
$$

$\gamma_{i j}$ is the $i j$ th cofactor of the matrix

$$
\mathbf{M}=\left|\begin{array}{lll}
x_{\xi} & x_{\eta} & x_{\zeta} \\
y_{\xi} & y_{\eta} & y_{\zeta} \\
z_{\xi} & z_{\eta} & z_{\zeta}
\end{array}\right|,
$$

and the Jacobian $J$ is the determinant of $M$. Central difference representation of the derivatives and relaxation techniques can be used for the solution of (9).

1.2b Grid control functions: The inherent smoothing properties of Laplace's equation ensures that, in the absence of boundary curvature, the mesh points are evenly spaced. However, near convex boundaries the grid points become more closely spaced, whilst near concave boundaries the mesh spacing is more sparse (figure 1). 

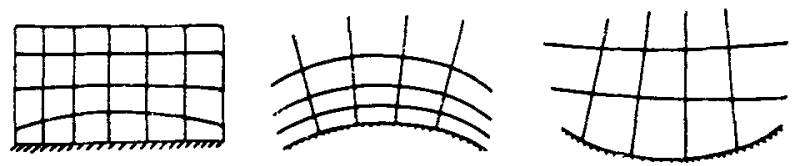

Figure 1. Mesh point spacing on concave and convex boundaries.

These properties are not always desirable for mesh generation where it is essential that the mesh near a boundary reflects the shape of that boundary. Control of mesh point spacing can be achieved by introducing source terms into Laplace's equation thus converting the system of equations into Poisson type. The source terms, often called grid control functions, can be so designed as to provide the flexibility required to control mesh point spacing.

Typical effects of the source functions $P$ and $Q$ in two dimensions are illustrated in figure 2. The major problem for automatic mesh generation is how to choose the control functions and, in some cases, the inherent parameters associated with them. Automatic methods have been developed (Sorenson \& Steger 1979) but one with a particularly beneficial formulation is that due to Thomas \& Middlecoff (1980). The basic idea behind this approach is to utilise the distribution of points on a boundary to compute the forcing functions which are then interpolated into the field. Using the elliptic equations, and assuming that all second derivatives transverse to the boundary are zero, yields the following equations

and

$$
P=-\left(x_{\xi}, x_{\xi \xi}+y_{\xi} y_{\xi \xi}\right) /\left(x_{\xi}^{2}+y_{\xi}^{2}\right), \text { along } \eta=\text { constant boundaries, }
$$

$$
Q=-\left(x_{\eta} x_{\eta \eta}+y_{\eta} y_{\eta \eta}\right) /\left(x_{\eta}^{2}+y_{\eta}^{2}\right), \text { along } \xi=\text { constant boundaries. }
$$

Hence, given a suitable boundary point distribution the control functions can be calculated to reflect this spacing in the field. The method readily extends into three dimensions, where the boundary points on the surfaces are used to generate the boundary values of the three control functions.

1.2c Other elliptic equations: The use of the Laplace and Poisson equations for mesh generation is now widespread. Several modifications to the basic system described in $\$ 1.2$ have been used (Thompson et al 1985). However, other different elliptic systems have been investigated and used successfully. Boerstoel (1988) has chosen to modify the Poisson formulation to include positive weight functions that are chosen to be inversely proportional to desired grid point distances along grid lines. Schwarz (1986) has extended the ideas on elliptic systems to include higher-order grid generation systems. Fourth-order (biharmonic) systems are implemented as a set
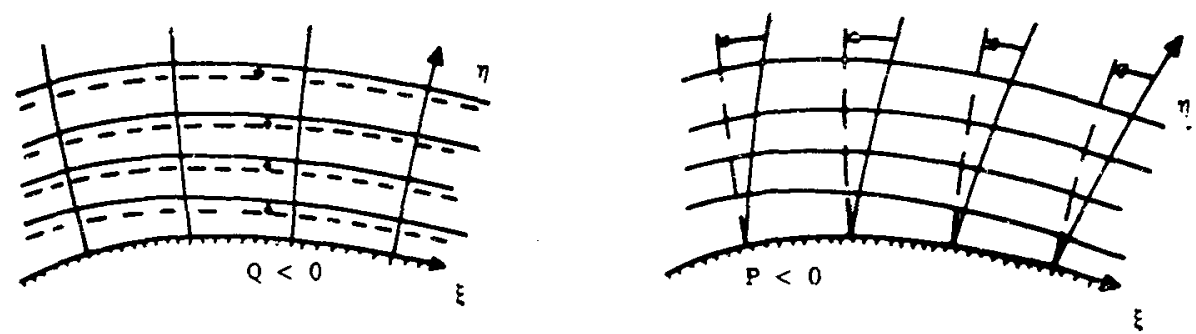

Figure 2. Effects of source functions $P$ and $Q$ on the mesh. 
of two second-order equations (Poisson's and Laplace's) whilst a sixth-order system is solved as a system of three second-order equations. The advantage of the higherorder systems is that they allow two and three boundary conditions to be specified for the fourth-order and sixth-order equations, respectively. This allows the geometry, the first or second grid lines from the boundary to be specified, thus assisting local grid control and mesh quality.

\subsection{Parabolic systems}

Parabolic mesh generation equations are usually derived from a suitable parabolisation of elliptic equations. The intention is to retain some of the desirable properties of the elliptic approach whilst capturing the computational efficiency of a marching technique. The diffusive effects inherent to the elliptic approach ensure that any discontinuities in the boundary data are smoothed. Control of the grid point distribution is achieved as for the elliptic approach and, with appropriate approximations, boundary values can be specified in the marching direction.

The parabolic approach has proved useful for a limited range of relatively simple geometries. Notable development work has been done by Nakamura (1982) and Edwards (1985). However, it is found that for complicated shapes the control of the diffusive effects, which in turn controls the ability of the equations to smooth discontinuities and rapid changes in boundary data, can prove difficult.

\subsection{Hyperbolic systems}

Mesh generation about a single body can be achieved using hyperbolic methods. The nature of the equations requires the grid to be marched away from the boundary data. The outer boundary cannot be fixed and thus the extent of the mesh must be controlled by the number of steps, the size of each step and parameters inherent to the system.

Steger \& Sorenson (1980) proposed a system of hyperbolic equations based on a volume-orthogonality scheme. The system

$$
\begin{aligned}
& x_{\xi} y_{\eta}-x_{\eta} y_{\xi}=V, \\
& x_{\xi} x_{\eta}+y_{\xi} y_{\eta}=0,
\end{aligned}
$$

is hyperbolic in $\eta$ provided $x_{\xi}^{2}+y_{\xi}^{2} \neq 0$. It is noted that (10b) is the condition for orthogonality. The function $V$ is used directly to control the mesh since it is a function which represents the volume (area) of each mesh cell. The quality of the mesh is determined by the function $V$. One approach is to choose a simple geometric shape which has the same arc length as the body to be meshed. An algebraic clustered mesh is then created with the required mesh point spacing. The volumes from such a mesh are then used in (10a). An example of a mesh around an aerofoil generated with the system (10) is shown in figure 3 . The discontinuity propagated from the trailing edge is clearly visible.

The advantages of a hyperbolic mesh generation procedure are that orthogonal grids which can offer good user control can be generated very rapidly. The disadvantages are that boundaries in the field, including the outer boundary cannot be specified and discontinuities in boundary data can be propagated into the field. This approach has been used successfully for simple geometries such as an aerofoil 


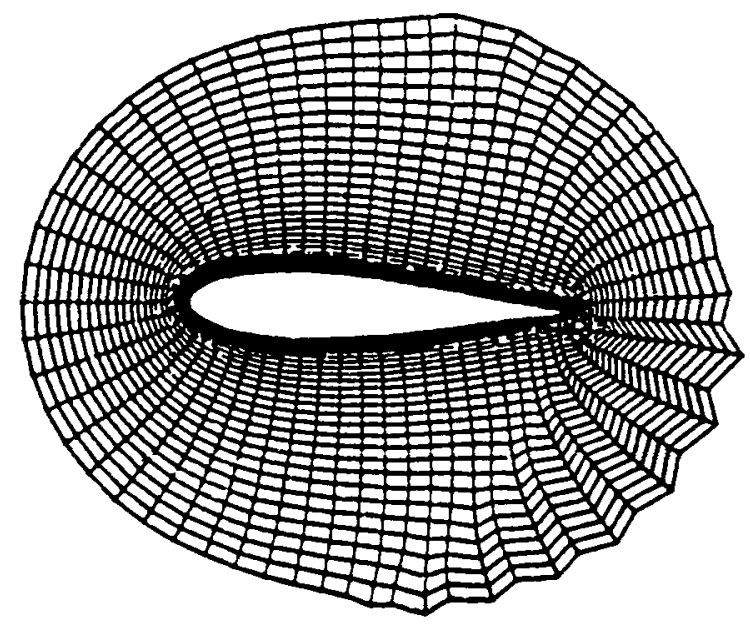

Figure 3. A mesh showing the propagation of a discontinuity generated from a hyperbolic system of equations.

and stacking planar meshes for applications to fuselage shapes (Steger \& Sorenson 1980).

\section{Conformal and orthogonal mapping}

\subsection{Introduction}

Conformal mapping has played a major role in the development of the understanding of fluid dynamics (Milne-Thompson 1968). The motion of a fluid which can be idealised as inviscid, irrotational and incompressible is governed by Laplace's equation for the velocity potential. The potential equation is invariant under a conformal mapping and hence the equation in the transformed space is often amenable to an analytic solution. In recent times with an emphasis on compressible flow, where the governing equations are both nonlinear and not invariant, the interest in conformal mapping has taken a secondary role to new techniques. Nonetheless, much use has been made of conformal mapping techniques since they can be used to conveniently map the infinite region of the flow exterior to, say, an aerofoil, onto a finite region, such as the interior of a circle. Furthermore, with a conformal mapping the form, in the mapped space, of the transformed equations takes on the simplest form for any mapping. In the solution of the Full Potential equation, these properties have been widely used.

In addition to these applications, new interest has arisen in conformal mapping techniques as the means of generating meshes. A geometrically complicated domain can be mapped into a simple domain within which a mesh is generated. Inverting the mesh into the physical domain then results in a computational mesh in the physical space. For example, if the boundaries of a flowfield are mapped onto a circle centred at the origin of a complex plane $\zeta=\rho e^{i \theta}$, the network of $\rho=$ constant and $\theta=$ constant lines, when mapped onto the physical plane, produce an orthogonal grid. Given a particular geometry, the search for an appropriate mapping is assisted by the many findings in the field of conformal mapping.

Many standard texts are available which discuss conformal mapping techniques 
(Kober 1952; Milne-Thompson 1968). With respect to the use of conformal mapping in mesh generation there have been a number of review papers (Ives 1975; Moretti 1980; Halsey 1986) and some detailed discussions on applications (Ives 1975; Halsey 1979).

The concept of a mapping is to define a transformation which takes a domain $D$, defined in the plane $(x, y)$, onto a domain $R$ on the plane $(\xi, \eta)$. The geometrical relationship between $D$ and $R$ is described by the components of the metric tensor $g_{i j}(i, j=1,2)$. From the definition of conformal mapping it follows that the components of the metric tensor are subject to the constraints

and

$$
g_{11}=g_{22},
$$

$$
g_{12}=0,
$$

and as such the mapping function $x(\xi, \eta)$ and $y(\xi, \eta)$ must satisfy the Cauchy-Riemann relations in the domain $R$,

$$
x_{\xi}=y_{\eta}, \quad x_{\eta}=-y_{\xi}
$$

and the condition of integrability yields

$$
\nabla^{2} x=0, \quad \nabla^{2} y=0 .
$$

Clearly from (12) it is not possible to fix both $x$ and $y$ on the boundary of $R$ for the solution of (13). One possibility is to fix the sides $\xi=$ constant of the rectangle $R$ with the value of the function of $x$ and from (12b) the value of the derivative $y_{\xi}$, while on the sides $\eta=$ constant the function $y$ and the derivatives $x_{\eta}$ are specified. Hence, it is apparent that in the case of conformal mapping it is not possible to specify the distribution of the grid points because of the constraints placed upon the metrics $g_{11}$ and $g_{22}$. A slightly more flexible approach can be achieved if the constraint of $g_{11}=g_{22}$ is relaxed and the relationship is taken as

$$
g_{11}=F^{2}(\xi, \eta) g_{22}, \quad g_{12}=0,
$$

where the specification of $F$, a dilatation function, permits a certain flexibility without the loss of orthogonality. Clearly, conformal mapping is a special case of (14) in which $F=1$. The analogous equations to (12) and (13) now become

and

$$
\begin{aligned}
& F x_{\xi}=y_{\eta}, \\
& x_{\eta}=-F y_{\xi},
\end{aligned}
$$

$$
\partial\left(F \partial x^{i} / \partial \xi\right) / \partial \xi+\partial\left(1 / F \partial x^{i} / \partial \eta\right) / \partial \eta=0, \quad x^{1}=x, x^{2}=y .
$$

This approach, however, is still restrictive on the imposition of boundary points and furthermore requires a suitable definition of $F$ (Arina 1986). Several papers have been devoted to investigations of conformal mappings based upon this numerical viewpoint (Trefethen 1986). However, perhaps the more familiar method is the use of classical closed form expressions.

\subsection{Classic mappings}

The Joukowski mapping

$$
z=\zeta+1^{2} / \zeta
$$


discovered by Joukowski (1910), and its associated Joukowski aerofoil profiles played a crucial role in the understanding of the mechanisms of lift and consequently in the theory of flight. The mapping of the $\zeta$-plane on the $z$-plane is equivalent to the successive transformations

$$
\zeta_{1}=1^{2} / \zeta, \quad z=\zeta+\zeta_{1}
$$

Given $\zeta$ and $\zeta_{1}$, the second of these reduces to simple addition on the Argand diagram. Using a circle in the $\zeta$-plane, this mapping can generate a two-parameter family of cusped profiles. Joukowski introduced the idea that a shifting of the centre of the circle is sufficient to produce an aerofoil having either camber or thickness or a combination of both.

The transformation (15) gives

$$
\mathrm{d} z / \mathrm{d} \zeta=1-1^{2} / \zeta^{2},
$$

so that $\mathrm{d} z / \mathrm{d} \zeta$ vanishes when $\zeta= \pm 1$ and therefore the representation ceases to be conformal in the immediate neighbourhood of these points. The point $\zeta=1$ is inside the circle and thus transtorms to a point inside the aerofoil. However, the point $\zeta=-1$ transforms into $z=-1$, the trailing edge. In view of this the transformation can be written

$$
(z-21) /(z+21)=[(\zeta-1) /(\zeta+1)]^{2} .
$$

In this form the Joukowski mapping produces a cusped trailing edge. However, Von Karman and Trefftz (1918) observed that the generalised form of (18), namely,

$$
(z-n 1) /(z+n 1)=[(\zeta-1) /(\zeta+1)]^{n}
$$

with $n=2-\lambda / \pi$, produces an aerofoil with a finite angle $\lambda$ at the trailing edge.

Equation (19) can be written in the form

$$
\left(z_{2}-s_{1}\right) /\left(z_{2}+s_{1}\right)=\left[\left(z_{1}-z_{1 t}\right) /\left(z_{1}+z_{1 n}\right)\right]^{1 / k}
$$

where $z_{1}$ is the aerofoil plane complex coordinates, $z_{2}$ the near-circle plane complex coordinate, $k=2-\lambda / \pi, z_{1 t}$ and $z_{1 n}$ are the two singular points placed in the $z$ plane at the trailing edge and at a point midway between the nose of the aerofoil and its centre of curvature, respectively, and the parameter $s_{1}$ is related to $z_{1 t}$ and $z_{1 n}$ though the relations $z_{1 t}=z_{s 1}+k s_{1}$ and $z_{1 n}=z_{s 1}-k s_{1}$.

A polar mesh with appropriate stretching in the radial direction generated around the near circle, when mapped back to the physical plane, produces a high quality mesh (figure 4).

Owing to its special role in theoretical aerodynamics, particular emphasis has been given here to the Joukowski type mapping and how it can be used to generate high quality meshes. However, there are many other mappings which play an important role in mesh generation. The parabolic mapping $\xi=z^{2}$ can be used to generate ' $C$ ' grids for aerofoils, whilst the Schwartz-Christoffel type of mappings can be applied to channels with arbitrary shape (Floryan 1983). For a full discussion on conformal mappings the reader is referred to a text on the subject.

\subsection{Applications in three dimensions}

Conformal mapping techniques, by their very nature, do not readily extend into three dimensions. In general, applications in 3 dimensions involve a conformal mapping 


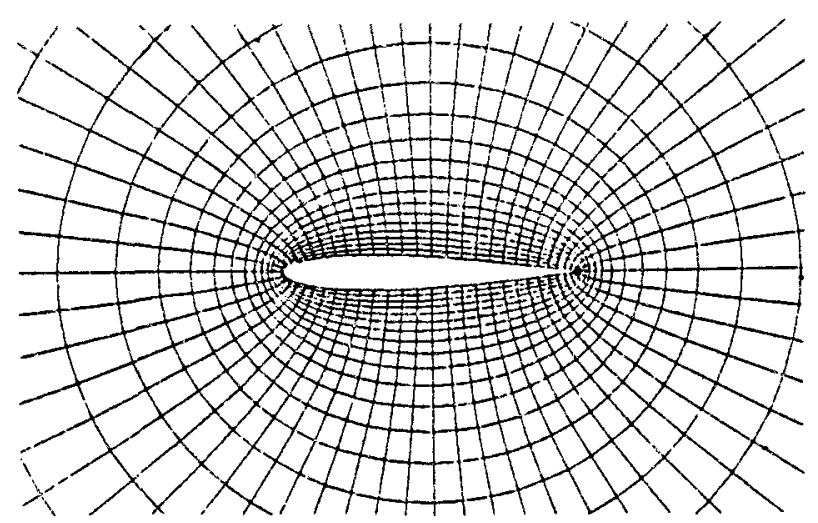

Figure 4. Mesh generated from the Karman-Trefftz transformation.

in 2 dimensions with some algebraic interpolation and stretching in the third direction. Recent work in the area of conformal mapping or near conformal mapping has been applied to complex 3-dimensional shapes. Baker (1986) has developed a transformation technique for a wing-body-tail-fin configuration.

\section{Algebraic mesh generation techniques}

\subsection{Introduction}

Algebraic mesh generation techniques provide a direct functional description of the transformation between the computational and physical domains. The classic algebraic method is conformal mapping in which closed form expressions of the transformation can be obtained and the mesh is orthogonal. However, these advantageous properties are counterbalanced by the inability to specify the position of the boundary points and the inherent two-dimensional nature of the method.

To relax these restrictions a class of mesh generation techniques has evolved which is based upon interpolation among boundaries and/or intermediate surfaces in the field. Such techniques are usually referred to as algebraic mesh generation methods.

In their simplest form such methods involve simple one-dimensional stretchings involving only boundary points. In more sophisticated formulations boundary points, slopes, curvature and interior control surfaces are included to extend the applicability of the algebraic method.

One of their major advantages is that the generation of a mesh is performed directly and is computationally very fast. Also, these techniques allow the explicit control of grid point spacing. Unfortunately, since the methods do not possess inherent smoothing properties discontinuities can arise in the mesh. However, a careful formulation of the algebraic procedures can usually alleviate such problems. It should also be noted that an important area of application of algebraic mesh generation is for use in interactive mesh systems.

\subsection{Multi-directional interpolation schemes}

If the shearing transformation is expressed for a 2-dimensional domain, then,

$$
R_{a}(\xi, \eta)=(1-\xi) R(0, \eta)+\xi R(1, \eta)
$$


expresses the interpolation $R_{a}$ derived by interpolating between the boundaries $\eta=$ constant. Similarly, for the boundary $\xi=$ constant, the interpolant,

$$
R_{b}(\xi, \eta)=(1-\eta) R(\xi, 0)+\eta R(\xi, 1)
$$

is obtained. The sum $R_{a}+R_{b}$ represents the interpolant, namely,

$$
R=R_{a}+R_{b}=(1-\xi) R(0, \eta)+\xi R(1, \eta)+(1-\eta) R(\xi, 0)+\eta R(\xi, 1) .
$$

This must recover the boundary values. However, if $\eta=0$ is substituted into (23) the result is

$$
R(\xi, 0)=(1-\xi) R(0,0)+\xi R(1,0)+R(\xi, 0) .
$$

Similarly unwanted interpolates are obtained on the other boundaries. In order to recover the correct boundary values it is thus necessary to construct a third interpolant $R_{c}$ which contains all the unwanted boundary interpolants. The form of this is

$$
\begin{gathered}
R_{c}(\xi, \eta)=(1-\xi)(1-\eta) R(0,0)+\xi(1-\eta) R(1,0)+ \\
+(1-\xi) \eta R(0,1)+\xi \eta R(1,1) .
\end{gathered}
$$

Thus the required interpolant is $R=R_{a}+R_{b}-R_{c}$ which can be expressed as

$$
\begin{aligned}
& R(\xi, \eta)=[(1-\xi) \xi]\left[\begin{array}{l}
R(0, \eta) \\
R(1, \eta)
\end{array}\right]+[R(\xi, 0) R(\xi, 1)]\left[\begin{array}{c}
1-\eta \\
\eta
\end{array}\right]- \\
& -[(1-\xi) \xi]\left[\begin{array}{ll}
R(0,0) & R(0,1) \\
R(1,0) & R(1,1)
\end{array}\right]\left[\begin{array}{c}
1-\eta \\
\eta
\end{array}\right] .
\end{aligned}
$$

This type of interpolation is called transfinite interpolation and the functions $\xi,(1-\xi)$, $\eta$ and $(1-\eta)$ are called blending functions. The simple ideas expressed here can be extended to include boundary slope data. In such cases, if the interpolant between sides of constant $\zeta$ is written as

$$
\begin{aligned}
\Pi_{\xi} f=f\left(\xi_{1}, \eta\right) \alpha_{1}(\xi)+\hat{\partial f} / \hat{\partial} \xi\left(\xi_{1}, \eta\right) \alpha_{2}(\xi)+ \\
+f\left(\xi_{2}, \eta\right) \alpha_{3}(\xi)+\partial f / \hat{c} \xi\left(\xi_{2}, \eta\right) \alpha_{4}(\xi)
\end{aligned}
$$

and between sides of constant $\eta$ as

$$
\begin{aligned}
\Pi_{\eta} f=f\left(\xi, \eta_{1}\right) \beta_{1}(\eta)+\partial f / \partial \eta\left(\xi, \eta_{1}\right) \beta_{2}(\eta)+ \\
+f\left(\xi, \eta_{2}\right) \beta_{3}(\eta)+\partial f / \hat{c} \eta\left(\xi, \eta_{2}\right) \beta_{4}(\eta),
\end{aligned}
$$

then the interpolant in the field can be written as

$$
\Pi_{\xi} \Theta \Pi_{\eta} f=\left(\Pi_{\xi}+\Pi_{\eta}-\Pi_{\xi} \Pi_{\eta}\right) f
$$

where $f=(x, y)$, and subject to the conditions

$$
\begin{aligned}
& x_{1}\left(\xi_{1}\right)=1, x_{1}\left(\xi_{2}\right)=0, \alpha_{1}^{\prime}\left(\xi_{1}\right)=0, x_{1}^{\prime}\left(\xi_{2}\right)=0, \\
& x_{2}\left(\xi_{1}\right)=0, \alpha_{2}\left(\xi_{2}\right)=0, \alpha_{2}^{\prime}\left(\xi_{1}\right)=1, \alpha_{2}^{\prime}\left(\xi_{2}\right)=0, \\
& x_{3}\left(\xi_{1}\right)=0, \alpha_{3}\left(\xi_{2}\right)=1, \alpha_{3}^{\prime}\left(\xi_{1}\right)=0, \alpha_{3}^{\prime}\left(\xi_{2}\right)=0, \\
& \alpha_{4}\left(\xi_{1}\right)=0, \alpha_{4}\left(\xi_{2}\right)=0, \alpha_{4}^{\prime}\left(\xi_{1}\right)=0, \alpha_{4}^{\prime}\left(\xi_{2}\right)=1,
\end{aligned}
$$

and similarly for $\beta$. Such formulations readily extend into three dimensions. 

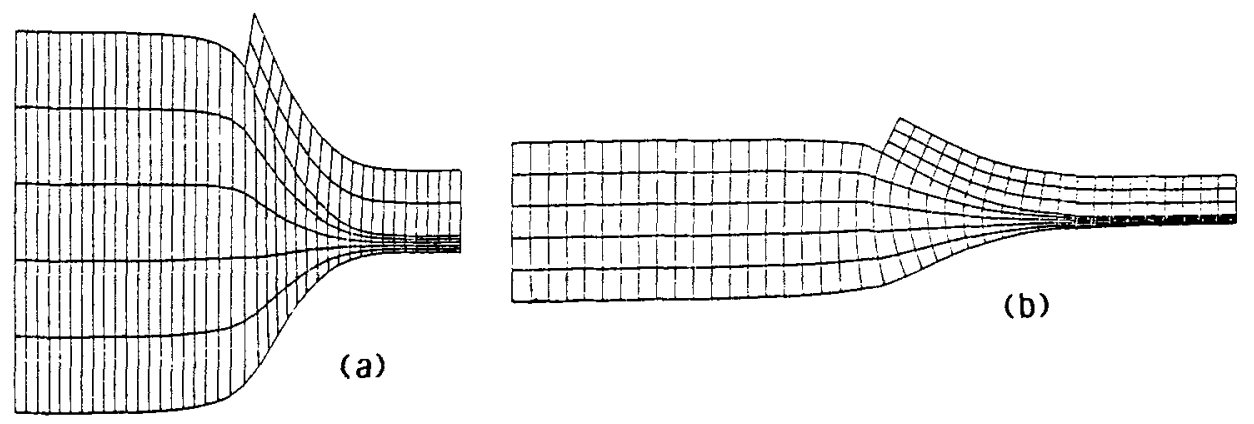

Figure 5. Mesh generated from uni-directional interpolation and for comparison of transfinite interpolation with control of boundary slopes.

More details of the approach are given in Gordon \& Hall (1973) and Eriksson $(1981,1982)$ and Faux \& Pratt (1979). Eriksson has particularly extended this method and has successfully constructed meshes for complicated domains.

As a point of interest, much of the early development work on transfinite interpolation was undertaken in the context of surface representation (Gordon \& Hall 1973).

The success of transfinite interpolation rests with the appropriate choice of the blending functions $\alpha$ and $\beta$ and the choice of values for the derivatives at the boundaries. Freedom to control the position of grid points is achieved by suitable adjustment of the inherent parameters.

A simple example of transfinite interpolation is given in figure $5(\mathrm{~J}$ Waddell, private communication). Although the scales in the two figures are slightly different it is clear that the grid lines in figure $5 \mathrm{a}$ are highly skewed at the boundaries. In this case linear interpolation has been used which, as already indicated, does not provide any control over the slope of the grid lines at the boundary. However, in figure $5 b$ a mesh is presented for a similar geometrical configuration which has been generated from transfinite interpolation with the incorporation of boundary slope data.

\section{Application of structured meshes - the multiblock approach}

\subsection{Introduction}

In general, structured mesh generation techniques produce a set of curvilinear coordinates throughout the domain. Unfortunately, for complex configurations the construction of an appropriate mesh from a single network of curvilinear coordinates is not practical. Thus the direct use of the techniques so far described is rather restricted. In practice, the use of these methods for realistic aerospace configurations is achieved in a blocked subdivision of the domain. Within each block the mesh is structured and continuity of slope, curvature etc. of grid lines across block faces can be achieved. Here a discussion of the basic concept of multiblock will be given.

\subsection{Basic concepts}

Central to the multiblock concept is the block subdivision of a domain. Here a block is defined as a geometrical domain which is topologically equivalent to a rectangle in two dimensions or a cube in three dimensions (figure 6). 

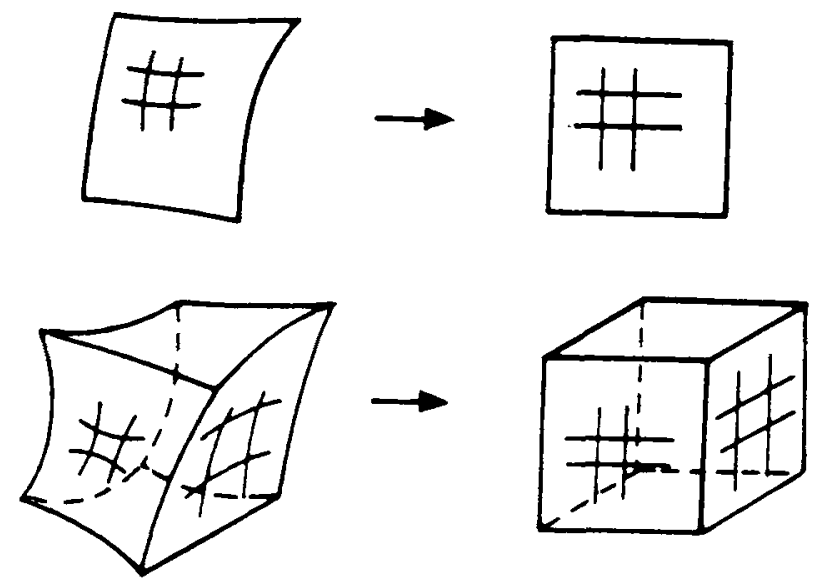

Figure 6. Regions in physical space are topologically equivalent to squares or cubes in the computational space.

A trivial example of a block subdivision of a domain around an aerofoil is given is figure 7 . Here, the mesh topology is a so-called $C$-mesh. The block subdivision for the mesh topology, shown in figure $7 \mathrm{a}$ is not unique. Two equally valid subdivisions are shown in figures $7 \mathrm{~b} \& \mathrm{c}$. In the case of figure $7 \mathrm{~b}$, the aerofoil boundary is restricted to one block side whilst in the second case the aerofoil boundary extends over one complete side and partially over two others. Either are possible subdivisions but for discussion purposes it will be assumed that only one type of boundary can exist on any block side. As will be demonstrated, this assumption does not in practice restrict the applicability of the multiblock approach, but does provide some simplification in the implementation.

Two other examples of block subdivision of the domain around an aerofoil are shown in figures 8 and 9. The block structure for a polar mesh can be reduced to a single block in which two opposite sides map onto each other. The Cartesian or $\mathrm{H}$-topology mesh around an aerofoil is given by six blocks suitably connected. Figures 7-9 show the block structures for the construction of the three basic mesh topologies around an aerofoil. These are rather trivial examples of the multiblock concept but they help to illustrate the basic ideas.

Consider now the more complicated geometry of two aerofoils, with rounded leading edges, arranged in a tandem configuration. It is possible in this case to generate
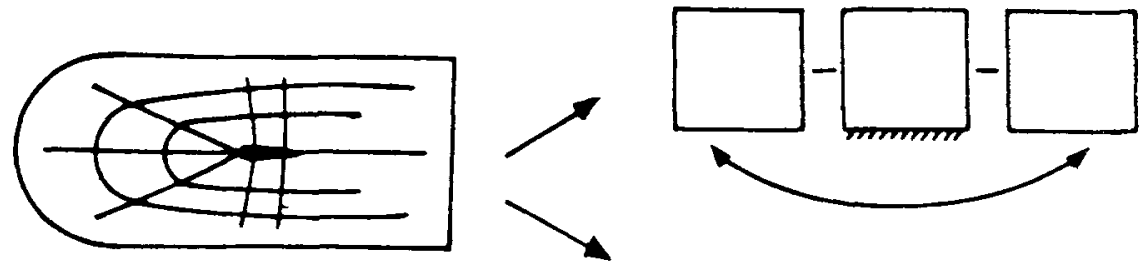

(b)

(a)

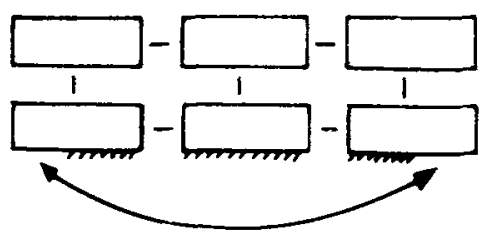

(c)

Figure 7. Block structure for a C-mesh topology. 

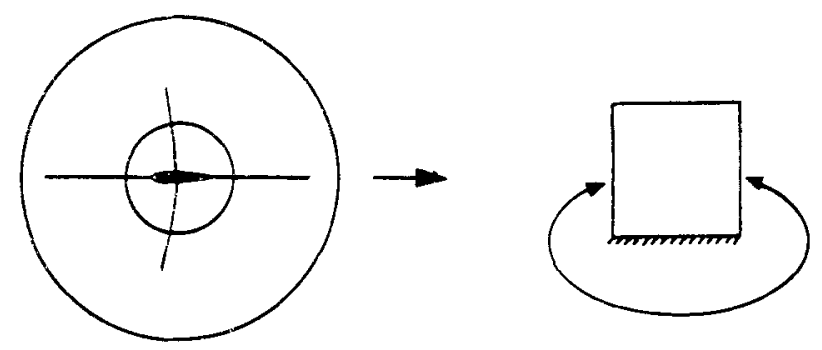

Figure 8. Block structure for an O(polar) mesh topology.

a mesh which effectively has one global coordinate system. A Cartesian mesh could be constructed local to each aerofoil in a way which is schematically shown in figure 10 , or, alternatively, the forward aerofoil could be favoured with a $C$-mesh but the aft-element would then have a Cartesian mesh (figure 11). On the grounds of mesh quality and flow sensitivity in the vicinity of the leading edges a Cartesian mesh local to the leading edge may be found to be unsuitable. Ideally, it can be argued, a $C$-mesh or a polar mesh is required to provide the necessary grid quality for accurate flow simulation. If this is the case, it is no longer possible to construct such mesh topologies and conserve a global curvilinear coordinate system. However, it is topologically possible to construct such meshes and the concept of a multiblock subdivision of the domain provides the appropriate mechanism.
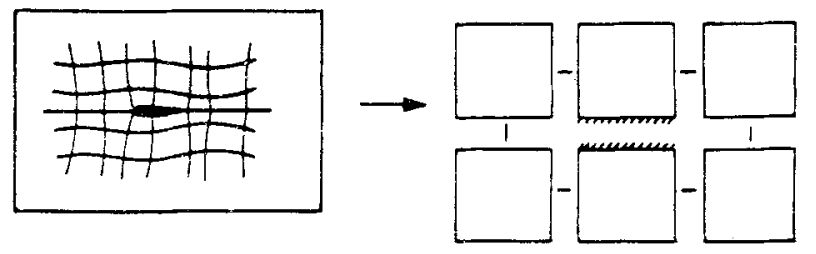

Figure 9. Block structure for an $\mathrm{H}$ (Cartesian) mesh topology.

Consider first the construction of a $C$-mesh local to each aerofoil. Figure 12 shows, in schematic form, one type of mesh which would satisfy this basic requirement. This is not the only mesh topology which would produce local $C$-meshes around the aerofoils, but it is suitable for the discussion. An analysis of this construction indicates that the equivalent block subdivision is made of 18 blocks. (In fact, it is possible to reduce this to 16 blocks, but, in order to ensure symmetry about the aerofoils a cut has been made from the leading edge of the first aerofoil to the upstream boundary.) The arrangement of these blocks and their connectivity is shown in figure 13 .

Now consider a mesh which has polar meshes locally around the aerofoils. Such a mesh is shown schematically in figure 14 with the equivalent block topology given

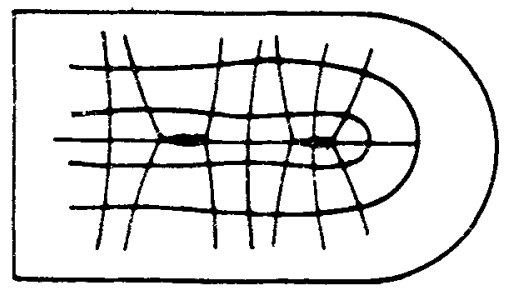

Figure 10. Cartesian mesh around both aerofoils.

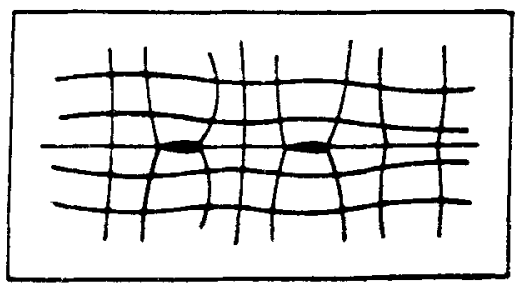

Figure 11. C-mesh around the leading aerofoil H-mesh around the second aerofoil. 


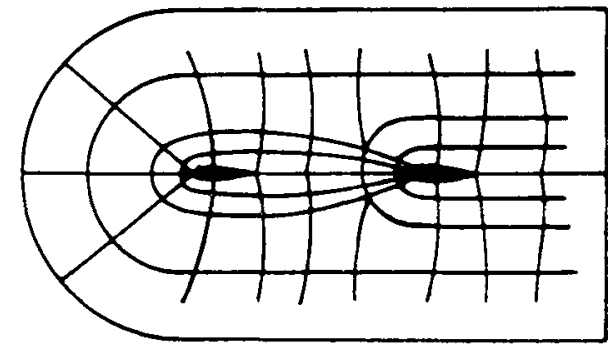

Figure 12. C-mesh topology constructed around both aerofoils.

in figure 15. Again, there is no unique way of decomposing the domain such that polar meshes are constructed.

These two examples illustrate that given a configuration, it is possible, using the concepts of multiblock domain decomposition, to construct mesh topologies which are ideally suited for each component of the configuration. The term 'component adaptive meshes' is used to describe this approach.

It is clear that the two mesh topologies shown in figures $12-15$ differ in the number of blocks and their arrangement or connectivities. Thus in a multiblocked environment it is necessary to define a way of describing the relative position of one block with respect to others i.e., just as in an unstructured mesh it is necessary to define an element connectivity matrix, it is necessary here to define a block connectivity matrix. In fact, at a block level the topology of a multiblocked mesh is equivalent to an unstructured quadrilateral mesh in the computational space.

This last observation is important. The block structure is completely independent of any coordinates in the physical space. The physical position of any computational block is not relevant in constructing the mesh topology. It is not appropriate to define a list of point coordinates and the connectivities between points. The form and information contained in the block connectivity data depends on the type of boundary conditions to be imposed at the interface between blocks. The types of block interface treatments will be discussed in detail in the next section. However, for clarity an example will be given here of the form of connectivity data required. One approach is to specify, for each side of every block,

(i) the type of boundary condition imposed, and

(ii) if appropriate. (a) the adjacent block number, (b) the adjacent side of the adjacent block, (c) the orientation of the coordinate system of the adjacent block relative to that of the current block.

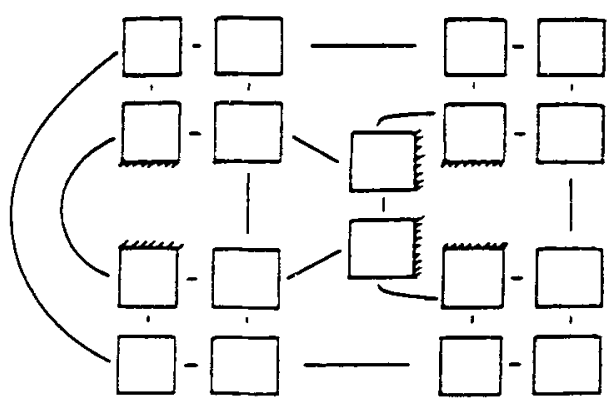

Figure 13. Block connectivity for the mesh topology shown in figure 12 . 


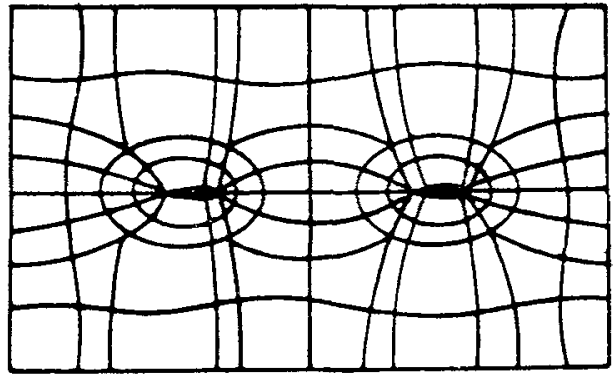

Figure 14. Polar mesh topology around each aerofoil.

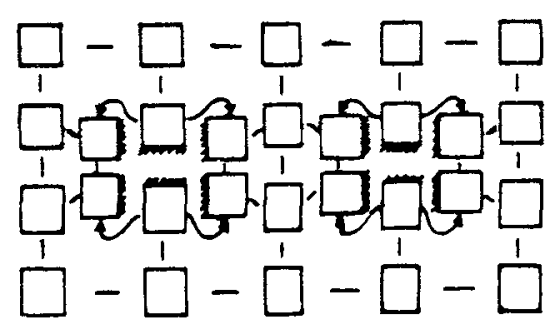

Figure 15. Block connectivity.

This is by no means the only way and other workers have chosen other approaches. This information, defined for each block, is collected together in what is termed the topology connectivity matrix. It is central to a mesh generation method based on the multiblock concept. This type of connectivity information is relevant to an approach in which grid points on block boundaries are treated in the same way as interior points. Before elaborating on this point a full discussion of block treatments will be given.

4.2a Solution procedures within a block and at block boundaries: 4.2a(i) Mesh generation within a block - It is clear from the discussion that each block has its own curvilinear coordinate system. This system is independent of those in the adjacent blocks. Hence, to generate a mesh within a block it is necessary to construct an algorithm which operates on a rectangular computational domain. With a suitable treatment of boundary points, the generation of the global mesh then involves a loop over all blocks.

If the mesh within each block is to be generated using a system of elliptic partial differential equations, such as those of (3), it is clear that for a block defined by $\left(\xi_{i}, \eta_{j}\right)$, $i=1,2, \ldots i_{\max }, j=1,2, \ldots j_{\max }$ in which the boundary points are assumed known, it is possible to solve for the interior points $\left(x_{i}, y_{j}\right), i=2, \ldots i_{\max }-1, j=2, \ldots j_{\max }-1$, without the requirement for information from adjacent blocks. This assumes a second order accurate central difference formulation for the derivatives in (3) and (4).

Thus, given a point $\left(\xi_{i}, \eta_{j}\right)$ the computational molecule for the solution of the elliptic equations at this point involves all immediate neighbours to the point. Hence assuming that the boundary points are known (equivalent to a Dirichlet condition), the interior points can be determined (figure 16).

A similar argument can be proposed if an alternative mesh generation technique

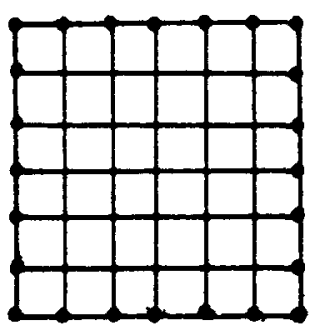

boundary points fixed

- solve for interior points

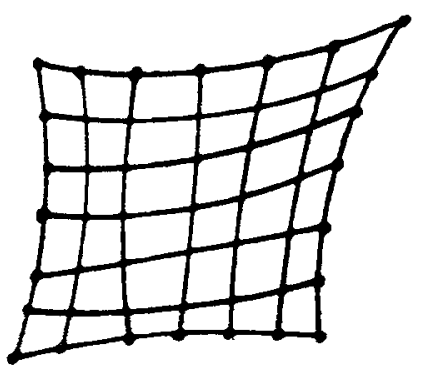

Figure 16. Solution procedure within a block 

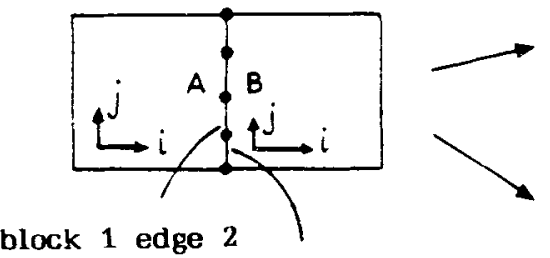

block 2 edge 1

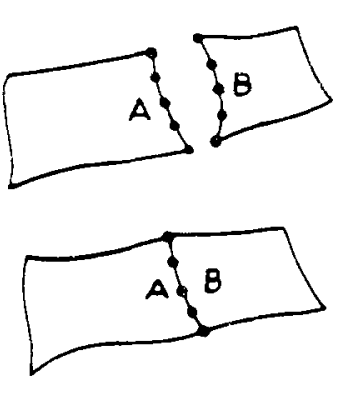

not allowed

points on common boundary are

coincident

Figure 17. Point correspondence on block edges.

is used. For example, transfinite interpolation is based upon a blended interpolation of boundary data into the interior of a domain. Clearly, given the boundary points of the blocks transfinite interpolation could be applied.

The basis for the ideas presented here is the assumption that the boundary points are either known or are computed by some other technique. There are several possible treatments for the boundary points.

4.2a(ii) Point continuity at block interfaces - Before discussing interface treatments, it is important to state a necessary condition which block boundaries must satisfy. Clearly, a computational mesh cannot contain holes or void regions. For this condition to be satisfied, it is necessary that, given two blocks which are adjacent and have a common edge in the block connectivity matrix, the grid points in physical space, which correspond to points on the edge, must be continuous (figure 17).

This is rather an obvious condition but one which should not be overlooked. Whatever treatment for the generation of points is designed for an edge it is necessary that the operations are commutative between common edges i.e. the treatment of the points on the edges in block 1 edge 2 must be equivalent to the treament of points on the block 2 edge 1 (figure 17).

4.2a(iii) Slope continuity at block interfaces-With fixed data points on block boundaries, it is possible to form a well-defined problem for the generation of grid points within a block. In some cases, fixed data points will correspond to points which define a geometrical object or boundary. In these cases, unless special treatments are applied, the grid lines extending from such boundaries will not possess special angular relationships with the boundary. A typical case is shown in figure 18 .

If a block boundary is adjacent to another block boundary, and the boundary points are fixed, then the resulting mesh will, in general, be discontinuous in gradients (and higher derivatives) across that boundary (figure 19). Clearly, in this case no information is provided to ensure any form of continuity and the mesh within each block is generated independently.

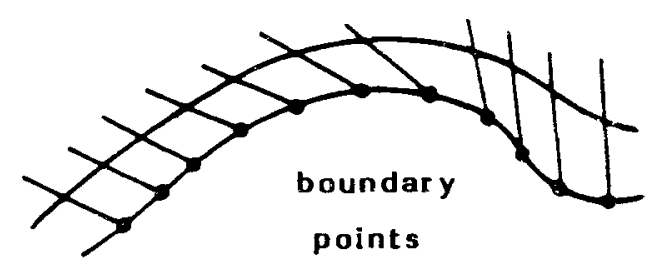

Figure 18. Grid points on boundary fixed. Angles of grid lines to the boundary are arbitrary. 


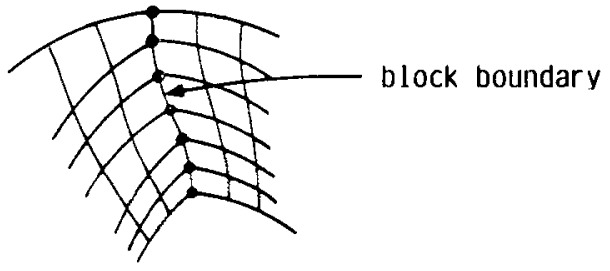

Figure 19. Grid lines discontinuous in slope across boundary.

It is possible to achieve slope continuity at block interfaces. As was indicated in $\$ 3 \cdot 2$, the transfinite interpolation method provides the mechanism by which slopes at boundaries can be specified. For elliptic systems, it is possible to manipulate the control functions to ensure definite slopes at boundaries. Provided grid lines and grid line intersection angles on both block edges which are common are defined to be equivalent, continuity of slope can be achieved.

4.2a(iv) Complete continuity at block interfaces - Any particular structured mesh generation scheme produces a mesh which is continuous to some definable level. The elliptic equations (3) generate curves continuous to degree two, i.e. there is continuity of slope and curvature. Similarly, interpolation schemes of the form (29) ensure continuity to a predetermined level. Complete continuity at block interfaces implies that the grid lines at block boundaries are as smooth, in the sense of differentiability, as lines interior to the blocks. Complete continuity at block interfaces can be achieved, but in this case the position of grid points on boundaries must, in general, evolve as part of the solution procedure for interior mesh points. The implication is that it is not possible to solve for grid points on a block-by-block basis, but rather the mesh in all blocks must be generated simultaneously.

Following this philosophy, the computational data necessary to generate the position of a point on a boundary must be of the same form as the information necessary to define the position of a point interior to a block. The position of an interior point, determined by solution of elliptic equations, requires information from direct neighbours (figure 20a).

For a point on a block boundary the same computational molecule is applicable but, in this case, some of the points in this molecule are contained in adjacent blocks (figures $20 \mathrm{~b}, \mathrm{c}$ ). For corner points the information is required from 3 adjacent blocks.

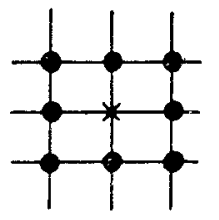

$x$-point in the interior of a block

a)

$X$ - point on an edge

- block boundary
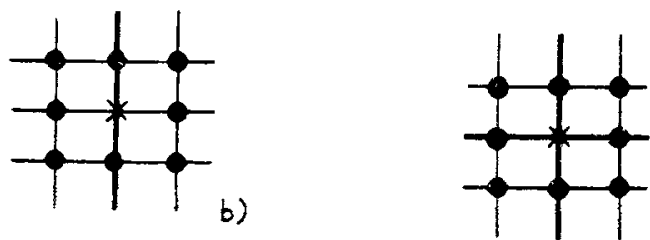

$X$-point at a

c)

block corner

Figure 20. Computational molecules for complete continuity at block boundaries. 


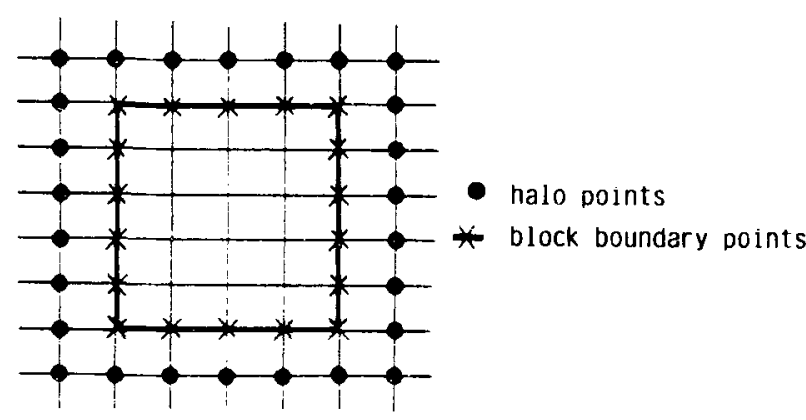

Figure 21. Block with halo points obtained from adjacent blocks.

Following this approach obviously leads to additional complexity in the data handling. No longer can a mesh in a block be derived from information contained only within that block. To ensure complete continuity the information related to a block must be augmented with information from other adjacent blocks. This information is, in general, effectively the halo of points surrounding the block (figure 21).

Any block of size $i=1, \ldots, i_{\max }, j=1, \ldots, j_{\max }$ is increased to size $i=1, \ldots, i_{\max }+2$, $j=1, \ldots, j_{\max }+2$. This increase in size provides the necessary space to accommodate the appropriate points from adjacent blocks. During the iterative solution routine the halo points are constantly updated when new values of halo points are derived.

The disadvantage of the halo system is that it increases the memory requirements. For large blocks the overheads are small but for small blocks the overheads are high relative to the block size. In fact, for a block consisting of a single point, a halo is required which contains 8 points. The main advantage is that having filled the halo with current values the solution routine acts on a rectangular domain with no special implementation required for boundary points.

\subsection{Review of the literature on multiblock}

The concept of a subdivision of the domain into rectangles (or cubes in 3 dimensions) within which curvilinear meshes are generated is a very popular method of applying structured meshes to complex geometrical shapes. There are many examples in the literature of this approach, although perhaps each differs in the fine details of the implementation. Coleman (1982) applied the method of 2-dimensional multicomponent geometries, whilst Miki \& Takagi (1984) applied the concept to 3-dimensional internal geometries. Roberts (1982, p. 465) discussed the method for aerodynamic shapes and Weatherill \& Forsey (1985) developed a method for 3-dimensional aircraft configurations. Other contributions in this area are to be found in the references (Vigneron \& Lejal 1984; Hauser \& Taylor 1986; Shaw et al 1986, 1988; Weatherill et al 1986; Thompson 1987; Boerstoel 1988; Hauser et al 1988; Weatherill \& Shaw 1988).

\subsection{Applications of the multiblock method}

4.4a Supersonic delta wing-body: The multiblock method described here (Weatherill \& Forsey 1985; Shaw et al 1986, 1988; Weatherill et al 1986; Weatherill \& Shaw 1988) has been used and tested for a variety of aircraft configuration. Two papers report detailed analysis of the performance applied to aircraft geometries (Baxendale 1988; Fulker \& Ashill 1988). 
Table 1. Different grid topologies used to model a wing-body aircraft configuration.

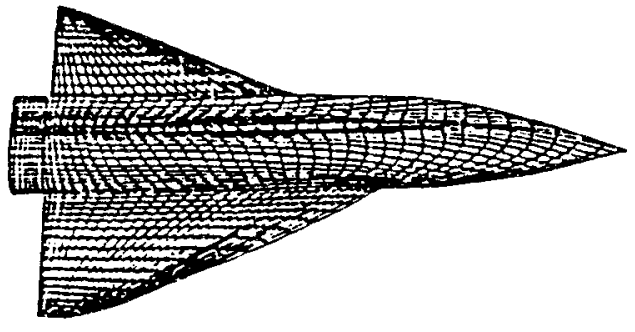

\begin{tabular}{|c|c|c|c|c|c|}
\hline Topology & $\begin{array}{l}\text { Structure } \\
\text { around } \\
\text { chordwise } \\
\text { section } \\
\text { of wing }\end{array}$ & $\begin{array}{l}\text { Structure } \\
\text { around } \\
\text { spanwise } \\
\text { section } \\
\text { of wing }\end{array}$ & $\begin{array}{c}\text { Structure } \\
\text { around body } \\
\text { cross-section }\end{array}$ & $\begin{array}{l}\text { Structure } \\
\text { around axial } \\
\text { sections of } \\
\text { forebody }\end{array}$ & $\begin{array}{c}\text { Number } \\
\text { of } \\
\text { blocks }\end{array}$ \\
\hline A & $\mathrm{C}^{\prime}$ & ${ }^{\prime} \mathrm{H}^{\prime}$ & 'H' & ${ }^{\prime} C^{\prime}$ & 24 \\
\hline B & ${ }^{\prime} \mathrm{C} '$ & ${ }^{\prime} \mathrm{H}^{\prime}$ & $\mathrm{O}^{\prime}$ & ${ }^{\prime} \mathrm{C}$ & 42 \\
\hline C & 'C' & ' $\mathrm{H}$ ' & $0^{\circ}$ & ' $\mathrm{H}$ ' & 106 \\
\hline
\end{tabular}

Fulker \& Ashill (1988) have studied three topologies for the simulation of the supersonic flow over a wing-body configuration (figure 22). Details of the accuracy of the flow simulations as functions of mesh point density and type of topology are given. Their study concludes that flow results are insensitive to grid topology, as judged by comparisons of overall features of pressure distribution, but one topology, namely $\mathrm{C}$ table 1 , was found to predict values of overall drag significantly different from the other two mesh topologies.

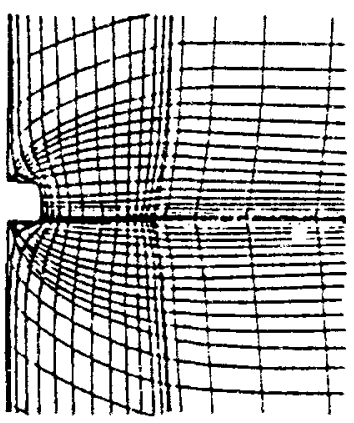

(a) topology $\mathrm{A}$

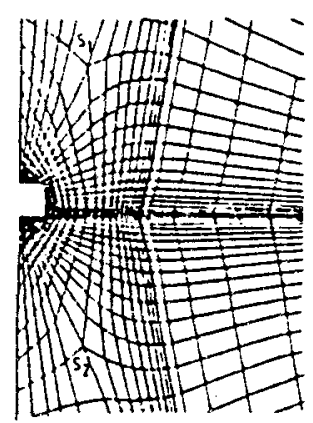

b) topologies $B$ and $C$

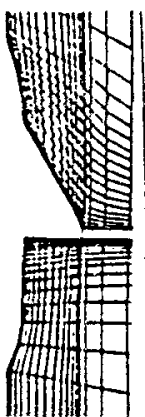

topology A

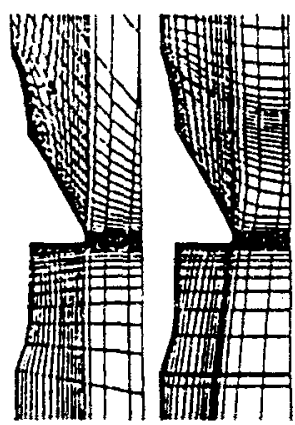

topology B

topelogy C
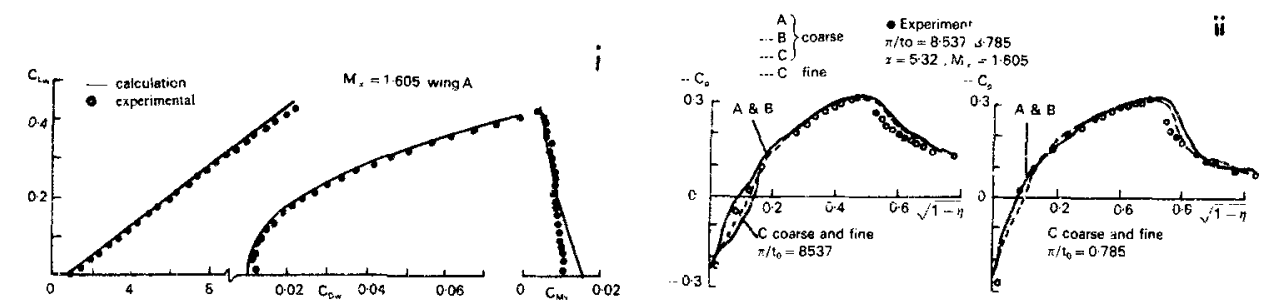

Figure 22. Delta wing. Results from Fulker \& Ashill (1988). (i) Overall lift, drag and pitching moment variations - comparison between theory and experiment. (ii) Typical detailed pressure distributions of various topologies. 


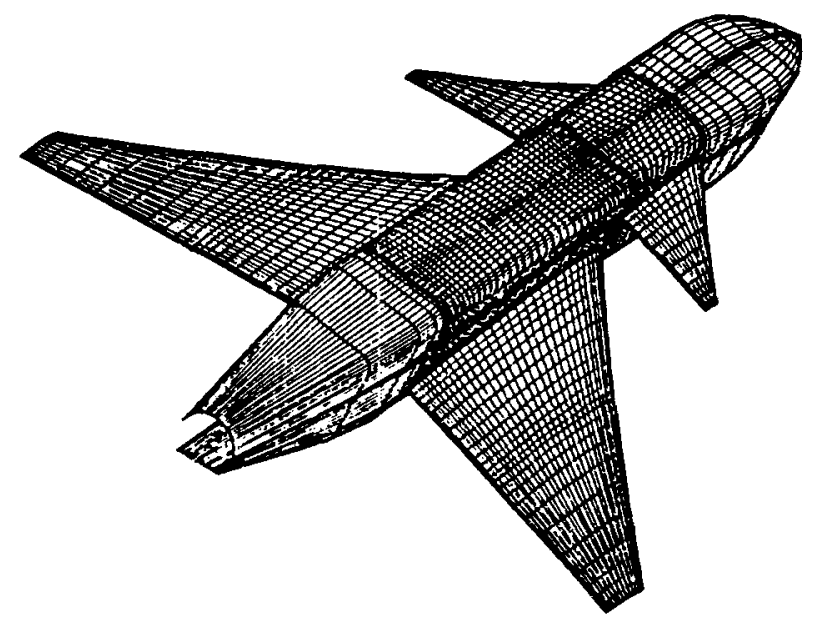

Figure 23. Surface grid. Wingbody-foreplane configuration (Baxendale 1988).

4.4b Wing-fuselage-foreplane configuration: In figure 23 a surface mesh for a foreplane configuration is given. A C-mesh topology is embedded local to each of the lifting surfaces, with a polar mesh around the body. An extensive number of flowfield calculations have been performed for a variety of foreplane positions (Baxendale 1988) and some of the results, in the form of forces and moments, are presented in figure 24 .

For build $\mathrm{A}$, the foreplane was mounted such that, at the body side, its trailing edge shared a common $x$-value with the leading edge of the wing. For builds $B$ and $C$, the foreplane was moved half a foreplane root chord forwards and backwards, respectively. The flow results presented in figure 24 show a good level of agreement with the experimentally measured data.

4.4c Wing combined with multiple bodies: Figure 25 shows a rather unusual aerodynamic configuration in which a prolate spheroid has been mounted on a wing.
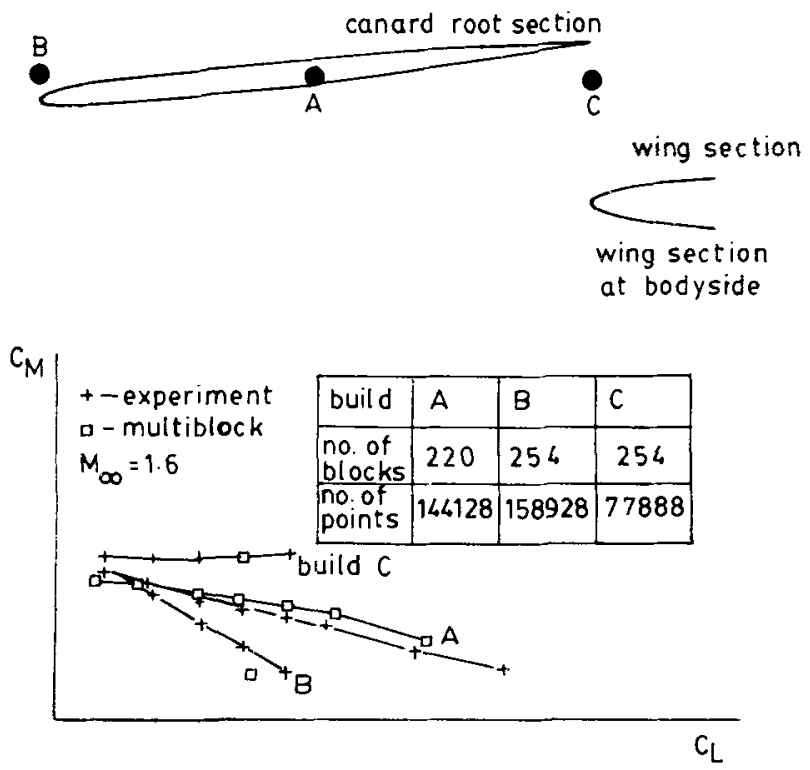

Figure 24. Flow results. (a) Alternative canard positions on bodyside; (b) effect of canard position on moment coefficient. 


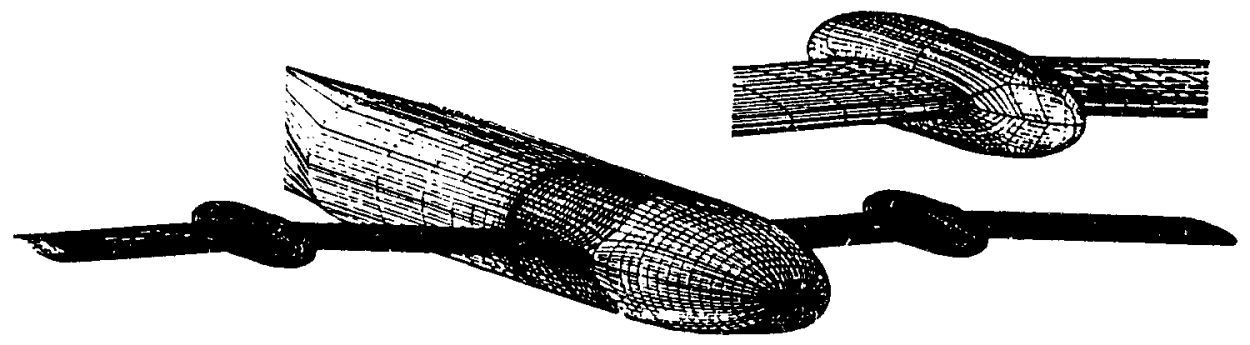

Figure 25. Wing with mid-mounted boom.

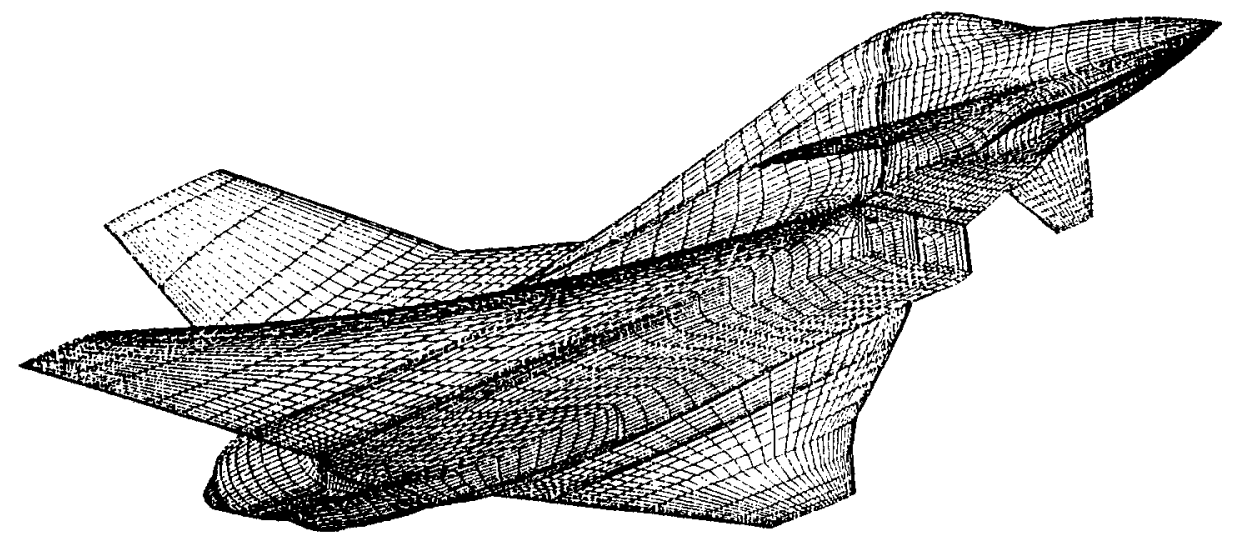

Figure 26. Complete surface grid for engine powered military aircraft (Shaw et al 1988).
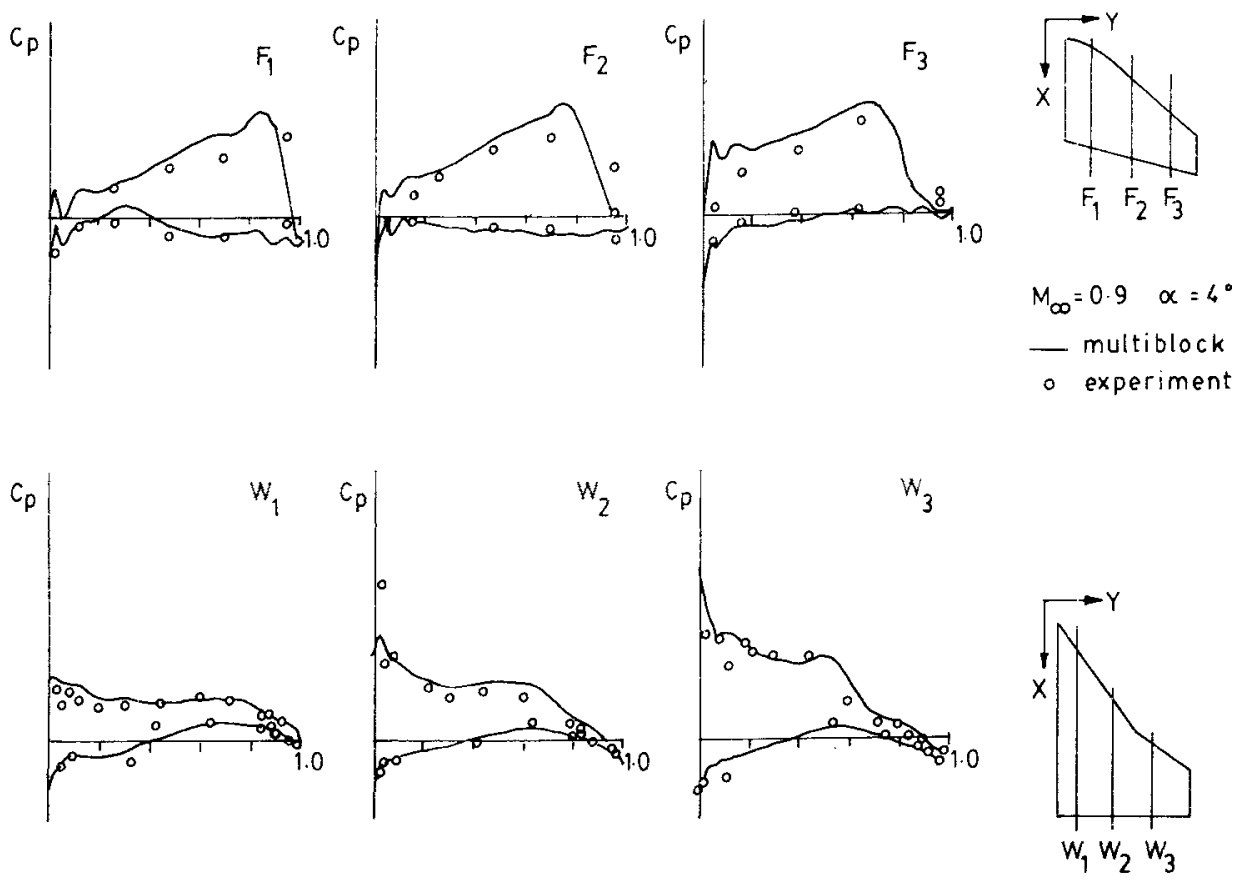

Figure 27. Flow results compared with experiment for the military aircraft. 
The topology used for this geometry is a $\mathrm{C}$-mesh around the wing and a spherical polar mesh around the mid-wing boom.

4.4d Military aircraft with propulsion units: A multiblock grid composed of 468 blocks has been generated for a modern military aircraft with twin engine intakes and afterbody nozzles (figure 26) (Shaw et al 1988). An O-mesh has been generated around the fuselage and afterbody nozzles, $\mathrm{C}$-meshes around the wing and foreplane and a Cartesian structure inside the sharp-lipped intake. Demonstration flowfield calculations have been performed for a range of freestream Mach numbers and engine mass flow ratios, and an example of such results is shown in figure 27.

These examples serve to illustrate the success which can be achieved from the multiblock approach.

\section{Automatic generation of unstructured meshes}

\subsection{Introduction}

The development of unstructured mesh generation schemes has advanced almost independently of the work on structured meshes. This is largeiy due to the fact that the nature of finite difference methods lends itself to a structured regular mesh, whilst unstructured meshes have been used traditionally with the finite element method. It is only recently that a new awareness of the common problems of mesh generation in both these areas has stimulated a closer dialogue between workers in these fields.

The basic difference between unstructured and structured meshes is in the form of data structure which most appropriately describes the meshes. A structured mesh of quadrilaterals or triangles consists of a set of coordinates and connectivities which naturally map into the elements of a matrix. Neighbouring points in the mesh in the physical space are the neighbouring elements in the mesh point matrix. However, for an unstructured mesh the points cannot be represented in such a manner and additional information has to be provided. For any particular point, the connection with: other points must be defined explicitly in the connectivity matrix.

The real advantage in the unstructured mesh is that the points and connectivities do not possess any global structure. It is possible to add and delete nodes and elements as the geometry requires or in a flow adaptivity scheme as flow gradients or errors evolve. Some techniques to generate unstructured meshes will be discussed.

\subsection{Advancing front technique}

One of the most generally applicable mesh generation techniques is based on the idea of the simultaneous mesh point generation and connectivity. Given a set of points, which defines a geometrical boundary or boundaries and a measure of the local spacing required within the domain, the method extends or advances the boundary connectivity into the field. Mesh points are generated and connected to other local points and in this way the mesh is advanced away from the boundaries (figure 28). The mesh point density is controlled by the user specified parameters which in the basic form, for a uniform mesh, can be a single value which represents the desired spacing throughout the domain.

The nature of the method makes it ideally applicable to the most complicated of 


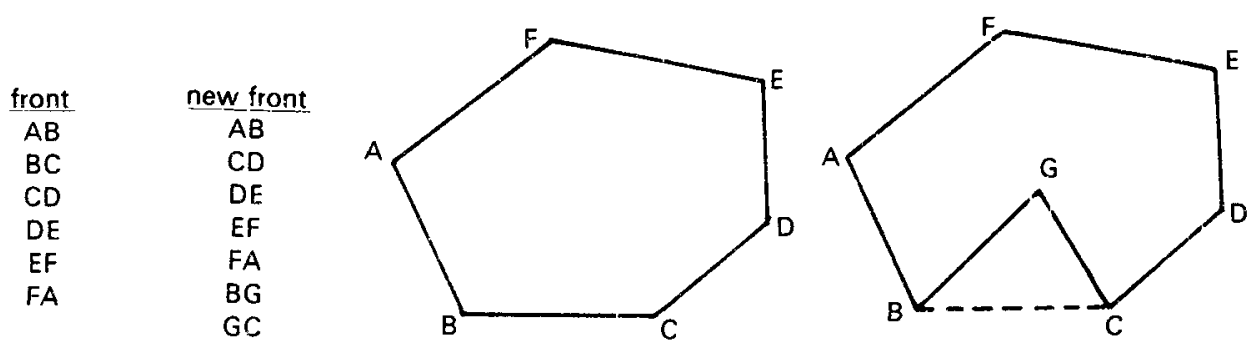

Figure 28. Basic concepts of the advancing front technique.

shapes and requires a minimal amount of data from the user. The approach, first discussed by George (1971), has been enhanced and extended into 3 dimensions by Peraire and coworkers (Peraire 1986; Peraire et al 1987). The approach of Peraire et $a$ is to define a mesh parameter $\delta$ which controls the local node spacing and a local stretching parameter $s$ and the local direction $\alpha$ of sketching. These parameters allow a mesh to be generated with variable sized elements, which are clustered in such a way that one-dimensional features in the solution can be captured in a very efficient manner.

Before starting to generate triangles inside a domain, the body points are created and the initial front, containing the information about all the boundary sides, is set up. The orientation of the boundary contours is such that the interior of the domain is always to the left. The initial front is defined by two integer vectors which have as many components as actual active sides. In one vector the number of the first node of each side is stored whereas the second node number is kept in the other vector. The generation front changes continuously throughout the process of triangulation and must be updated whenever a new element is formed. Thus the front changes during the triangulation process and reduces to zero when the end of the triangulation is reached. Every time a triangle is generated its sides are added as new entries in the front.

In the process of generating a new triangle, the following steps are involved.

(1) A front side, with node A and B, to be used as the base for a triangle is selected. With distance $\delta_{1}$ from points $\mathrm{A}$ and $\mathrm{B}$, a point $\mathrm{C}$ is chose. $\delta_{1}$ is chosen as

$$
\delta_{1}= \begin{cases}\delta, & \text { if } 0.55 \times \overline{\mathrm{AB}}<\delta<2 \times \overline{\mathrm{AB}}, \\ 0.55 \times \overline{\mathrm{AB}}, & \text { if } 0.55 \times \overline{\mathrm{AB}}>\delta, \\ 2 \times \overline{\mathrm{AB}}, & \text { if } \delta>2 \times \overline{\mathrm{AB}}\end{cases}
$$

(2) Find all the nodes which belong to any of the current front sides and lie inside the area determined by the circle (centre $\mathrm{C}$ and radius $r=3 \times \overline{\mathrm{AB}}$ ) and the points $\mathrm{A}$ and $\mathrm{B}$. Order these nodes $\left(a_{i}, i=1, n\right)$ according to their distance from the point $\mathrm{C}$, in such a way that the first node in the list is the closest to $C$.

(3) Decide whether or not point $\mathrm{C}$ is added to the list. If

$$
\begin{aligned}
& \overline{\mathrm{Aa}_{1}}<1.5 \times \delta_{1}, \\
& \mathrm{Ba}_{1}<1.5 \times \delta_{1},
\end{aligned}
$$

then $\mathrm{C}$ is not included. Otherwise $\mathrm{C}$ is placed in the first position of the list and the other nodes are shifted by one place. 


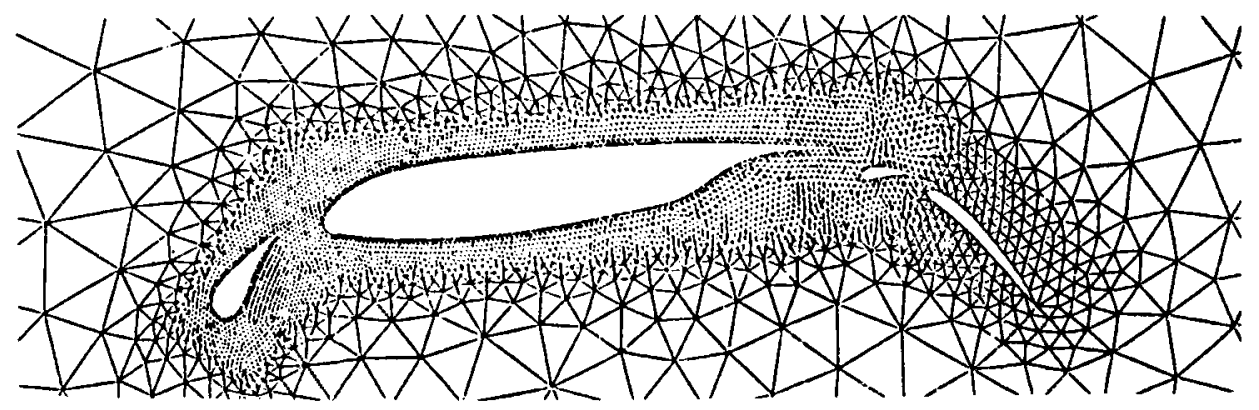

Figure 29. Example of a mesh generated by the advancing front technique (Peraire $e t$ al 1987).

(4) Determine the connecting point $a_{j}$. This is taken to be the first node in the list that satisfies the two following validity requirements.

(i) the interior of the triangle $\mathrm{ABa}_{j}$ does not contain any of the remaining nodes in the list (excluding $\mathrm{C}$ ).

(ii) The segment line $a_{i} M(M$ midpoint of $A B)$ does not intersect any of the existing sides of the front.

(5) Form and store the new element. If $\mathrm{C}$ is chosen as the connecting point a new node is created.

This approach can be extended to a non-uniform distribution of $\delta$ and also the inclusion of element stretching. Details can be found elsewhere (Peraire 1986). Two examples taken from Peraire et al (1987) are given in figures 29 and 30.

This technique has been extended into three-dimensions and has been successfully used to mesh complete aircraft configurations (Peraire et al 1987).

\subsection{Point generation followed by connection}

This approach, in principle, involves two distinct parts. First, a set of points is defined which covers the domain, and second, these points are connected to form a mesh. Although all mesh generation techniques effectively perform these tasks it is convenient to define such methods which emphasize connectivity techniques.
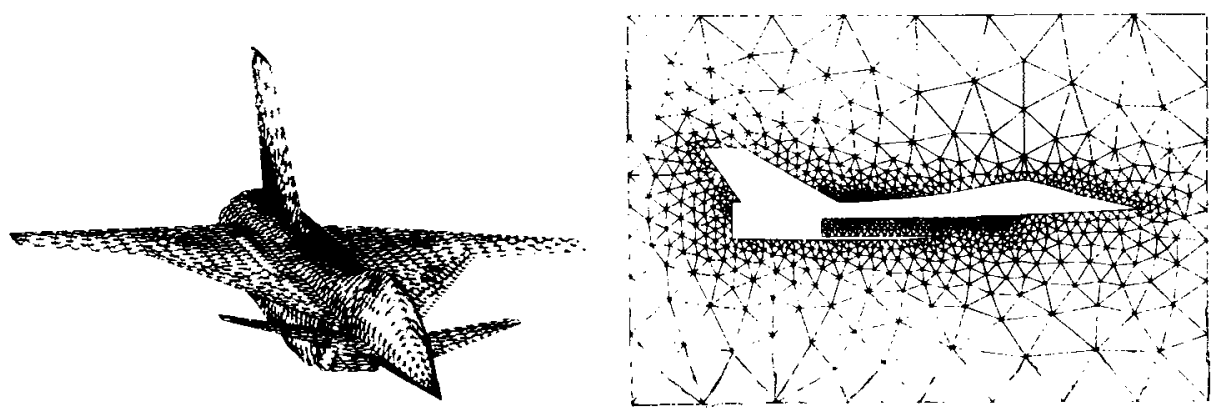

Figure 30. Mesh from the advancing front technique (Peraire et al 1987). 
Given a set of points in the plane it is a trivial task for the brain to connect them to obtain a triangulation. However, the development of algorithms to perform this task is not trivial. Early attempts employed ad hoc rules to determine the appropriate connections of the points. In practice, the implementation of these rules involved a large number of checks and search procedures which did not always lend themselves to application in 3 dimensions (Watabayashi \& Galt 1986).

The requirement to base an algorithm on a sound mathematical basis cannot be overemphasised and more recent work in this area has largely centred around the Voronoi construction. Section 6 is devoted to a discussion of this technique.

\subsection{Domain decomposition}

This class of methods involves the iterative subdivision of a domain into small units. The number of subdivisions is usually based on fixed criteria, such as the need to ensure regular subdivisions close to given boundary data. Often these routines, to ensure efficiency, are based around appropriate data structures. Examples of this kind are the quadtree and octree methods for 2- and 3-dimensional domains, respectively.

Albone (1988) makes use of this basic idea in his Feature-Associated Mesh Embedding (FAME) technique. Assume that a set of points is given which define, for example, an aerofoil and which are contained within a quadrant defined by 4 points. This quadrant is further subdivided into four quadrants. For each quadrant, a test is made to determine whether there are any surface mesh points in that quadrant for which $\delta<\Delta_{1} d$, where $\delta$ is a typical spacing of the surface mesh points, $\Delta_{1}$ is the mesh length in the original block and $d$ is a control parameter (taken as $\sqrt{2}$ for 2-D flows). If a quadrant contains such points then that quadrant is subdivided by a factor of two in each coordinate direction. If no such points occur then the quadrant is not subdivided. For each quadrant, up to 4-blocks can be created and each has a mesh length of $\Delta_{2}=\frac{1}{2} \Delta_{1}$. This subdivision process is continued and further embedding is made for which $\delta_{2}<\Delta_{2} d$. This strategy then ensures that local grid clustering is compatible with the predefined surface mesh point distribution. These points can then be used for triangulation (via Delaunay) or as Albone chooses to provide an underlying mesh for flow simulation.

\subsection{Other methods}

There are approaches which do not naturally fall into the category of structured or unstructured but more correctly are combinations of both techniques.

The idea of generating meshes appropriate to different components of a configuration where the meshes overlap has been investigated (Steger et al 1983; Benek et al 1985). The approach involves transferring information from one mesh to another within the overlapping regions by the use of interpolation techniques.

Nakahashi \& Obahashi (1987) have utilised curvilinear meshes local to bodies but used regions of unstructured meshes to connect these meshes. This is similar to the approach discussed in $\S 7$, where the aim has been to capitalise on the advantageous properties of the structured and unstructured approaches.

Finally, in this section it is appropriate to reference the many mesh generation techniques which have been developed for use with the finite element method (Thacker 1980; Sheppard 1988). 


\section{Voronoi regions and the Delaunay triangulation}

\subsection{Introduction}

A mesh is defined as a set of points $P_{i}$ and an appropriate connection between the points. The points act as reference positions within the field at which flow variables are to be computed and the connections between points act as pathways for transferring information around the computational domain. In structured mesh generation techniques the connections between points are defined through the curvilinear coordinate system. In unstructured mesh generation the connectivities have to be defined. This additional effort required is, however, counterbalanced by the additional flexibility achieved with an unstructured mesh. In unstructured mesh generation, unlike structured mesh generation, the problem of mesh point generation and connectivity of points decouple. This property opens new avenues of investigation into mesh generation.

The generation of unstructured meshes now becomes that of

(i) specifying the coordinates of the points, and

(ii) connecting the points such that the resulting mesh is appropriate for a discretisation of the governing flow equations.

Earlier chapters have discussed techniques for point generation and these ideas although general, e.g. conformal, elliptic partial differential equations, are usually applied to specific shaped domains. (In fact, this is the case since structured mesh generation involves a mapping process.) As already remarked, the difficulty with such techniques is when the domain does not readily map to a rectangle or cube. However, given a relatively simple geometry, e.g. a wing, pylon, fuselage, nacelle etc., it is not difficult to adopt one of these methods and compute a high quality mesh around one such component. Thus, it can be argued, the first part of unstructured mesh generation, namely, coordinate point definition for individual components is not difficult. A complex shape can be broken down into a subset of parts for which mesh generation techniques can be applied to produce a good point distribution appropriate for that part. However, the union of these point sets will not, in general, be such that a curvilinear coordinate network can be derived and hence the points must be connected in some other way.

At this stage, it is possible to argue that since the sub-meshes will overlap it would be possible to interpolate information from one mesh to another. This approach has been investigated (Steger et al 1983; Benek et al 1985). However, an alternative approach, and that followed by Jameson, Baker and Weatherill (Weatherill 1985, 1988; Jameson et al 1986; Baker 1987, 1988; Jameson \& Baker 1987), is to reconnect the points in a consistent manner thus producing an unstructured mesh of triangles. In this way interpolation between points can be avoided. Following this approach the problem becomes one of establishing a method which can connect arbitrary points to form a consistent triangulation. A systematic approach to achieve this dates back to 1850 and is credited to Dirichlet (1850).

\subsection{Voronoi neighbourhoods and Delaunay triangulation}

Dirichlet (1850) proposed a method whereby a given domain could be systematically decomposed into a set of packed convex polygons. Given a set of points in the plane, 
the Dirichlet tessellation is the construct which assigns to each point a territory that is the area of the plane closer to that point than to any other point in the set. This tessellation of a closed domain results in a set of non-overlapping convex polygons, called Voronoi regions, covering the entire domain. This definition readily extends to higher dimensions.

This basic idea, although first described by Dirichlet, has been subsequently redisco vered by various workers in many fields and the Dirichlet regions are described under a variety of names: Theissen regions (meterology), Wigner-Seitz cells (solid state physics) and Voronoi diagrams (computational geometry). An extensive discussion on the application of Voronoi diagrams is given in Green \& Sibson (1978).

If a set of points is denoted by $\left\{P_{i}\right\}$, then the Voronoi region $\left\{V_{i}\right\}$ can be defined as

$$
\left\{V_{i}\right\}=\left\{p:\left\|P-P_{i}\right\|_{i}<P-P_{j} \|, \forall_{j \neq i}\right\}
$$

i.e., the Voronoi region $\left\{V_{i}\right\}$ is the set of all points of $p$ that are closer to $P_{i}$ than to any other point. The sum of all points $p$ forms a Voronoi polygon.

From this definition, it is clear that, in two dimensions, the territorial boundary which forms a side of a Voronoi polygon must be midway between the two points which it separates and is thus a segment of the perpendicular bisector of the line joining these two points. If all point pairs which have some boundary in common are joined by straight lines, the result is a triangulation of the convex hull of the data points. This triangulation is known as the Delaunay triangulation of the set of points $\left\{P_{i}\right\}$.

It is apparent that for each triangle there is an associated vertex of the Voronoi diagram which is at the circumcentre of the three points which form the triangle. In the other words, each Delaunay triangle has a unique vertex of the Voronoi diagram and no other point within the Voronoi structure lies within the circle centred at this vertex. Figure 31 shows a schematic of a Voronoi diagram and associated Delaunay triangulation of a small set of points. In three dimensions, the territory of each data point is a convex polyhedron and each vertex of the Voronoi structure is at the circumcentre of a sphere defined by four points which describe the Delaunay tetrahedron.

A survey of computational methods to construct the Voronoi diagram and Delaunay triangulation is given in the paper by Green \& Sibson (1978). Recent noteworthy papers which describe efficient algorithms are those of Watson (1981), Bowyer (1981) and Fortune (1985). The algorithm used here is based on the work of Bowyer (1981).

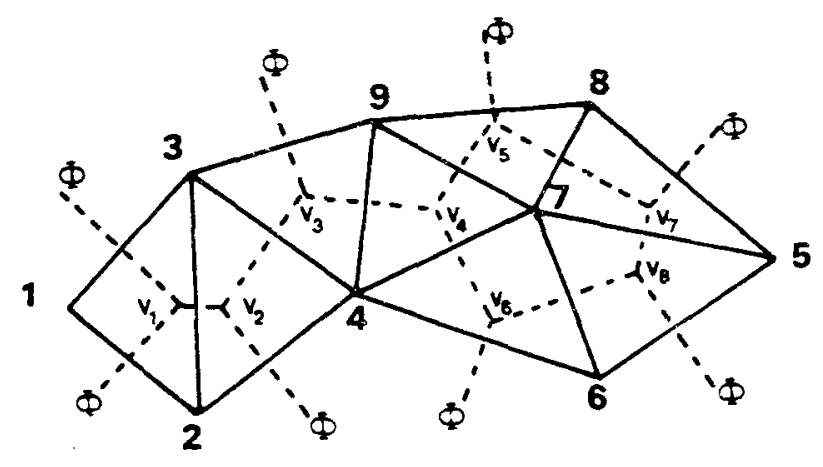

Figure 31. Voronoi regions and associated Delaunay triangles of the set of points $i(i=1,9)$. 
Table 2. Data structure for the triangulation shown in figure 31 .

\begin{tabular}{lllllll}
\hline Vertex & Forming points & \multicolumn{2}{c}{ Neighbouring vertices } \\
\hline 1 & 1 & 2 & 3 & 2 & $\Phi$ & $\Phi$ \\
2 & 2 & 3 & 4 & 1 & 3 & $\Phi$ \\
3 & 3 & 4 & 9 & 2 & 4 & $\Phi$ \\
4 & 4 & 7 & 9 & 3 & 5 & 6 \\
5 & 7 & 8 & 9 & 4 & 7 & $\Phi$ \\
6 & 4 & 7 & 6 & 4 & 8 & $\Phi$ \\
7 & 5 & 7 & 8 & 5 & 8 & $\Phi$ \\
8 & 5 & 6 & 7 & 5 & 8 & $\Phi$ \\
\hline
\end{tabular}

\subsection{Delaunay triangulation algorithm}

It is possible to completely describe the structure of the Voronoi diagram and Delaunay triangulation by constructing two lists for each Voronoi vertex; a list of forming points of a Voronoi vertex (the forming points define a Delaunay triangle) and a list of the neighbouring Voronoi vertices. In $n$ dimensions, each vertex has $n+1$ forming points and $n+1$ neighbouring vertices. As an example, table 2 contains the vertex structure for the points shown in figure 31 .

The Delaunay algorithm is a sequential process; each new point is introduced into the existing structure, which is broken and then reconnected to form a new Delaunay triangulation.

The details of the algorithm will be presented in a step-by-step manner for a triangulation in two dimensions.

\section{Step 1}

Define the convex hull which will contain all points. Specify four points together with the associated Voronoi diagram structure. Figure 32 indicates a typical start-up layout with the associated Voronoi diagram structure and table 3 describes the corresponding data structure.

Vertices 1,2,3,4 are not strictly defined, since they lie outside the convex hull and therefore do not possess three points and do not have three neighbouring vertices.

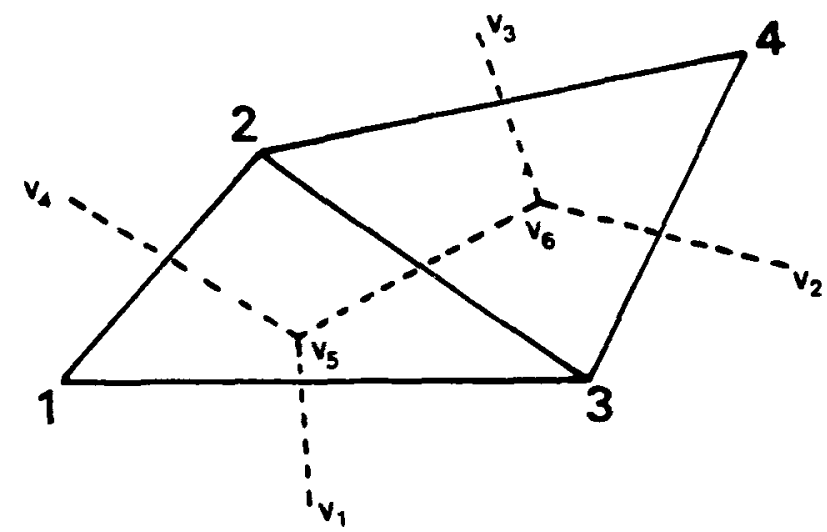

Figure 32. Initial points which define the convex hull with associated vertices. 
Table 3. Data structure for the triangulation shown in figure 32 .

\begin{tabular}{lrrrrrr}
\hline $\begin{array}{l}\text { Vertex Voronoi } \\
\text { diagram }\end{array}$ & Forming points & \multicolumn{2}{l}{ Neighbouring vertices } \\
\hline 1 & -10 & 1 & 3 & -10 & -10 & 5 \\
2 & -10 & 3 & 4 & -10 & -10 & 6 \\
3 & -10 & 2 & 4 & -10 & -10 & 6 \\
4 & -10 & 1 & 2 & -10 & -10 & 5 \\
5 & 1 & 2 & 3 & 1 & 4 & 6 \\
6 & 4 & 2 & 3 & 5 & 2 & 3 \\
\hline
\end{tabular}

Default values of -10 for the missing forming points and neighbouring vertices have been used to indicate that these are null vertices.

Step 2

Introduce the new point anywhere within the convex hull $\{1,2,3,4\}$.

Step 3

Determine all vertices of the Voronoi diagram to be deleted. A point which lies within the circle, centred at a vertex of the Voronoi diagram and which passes through its three forming points, results in the deletion of that vertex. This follows from the definition of a Voronoi polygon.

Step 4

Find the forming points of all the Voronoi vertices. These are the contiguous points to the new point.

Step 5

Determine the neighbouring Voronoi vertices to the deleted vertices which have not themselves been deleted. These data provide the necessary information to enable valid combinations of contiguous points to be constructed.

Step 6

Determine the forming points of the new Voronoi vertices. The forming points of the new vertices must include the new point together with two other points which are contiguous to the new point and form an edge of a neighbouring triangle (these are the possible combinations obtained from step 5).

Step 7

Determine the neighbouring Voronoi vertices to the new Voronoi vertices. Following step 6, the forming points of all new vertices have been computed. For each new vertex, perform a search through the forming points of the neighbouring vertices, as found in step 5, to identify common pairs of forming points. When a common combination occurs, then the two associated vertices are neighbours of the Voronoi diagram.

\section{Step 8}

Reorder the Voronoi diagram data structure, overwriting the entries of the deleted vertices.

\section{Step 9}

Repeat steps 2-8 for the next new point. 
With the exception of step 3, all the operations described are of a local nature and can be carried out in an operation count independent of the total number of points within the current structure. A naive search for vertices of the Voronoi diagram to be deleted would amount to $O(N)$ operations per point and thus to $O\left(N^{2}\right)$ operations for the total workload. An obvious improvement is to identify any one vertex that is to be deleted and then perform a tree search through the neighbouring Voronoi diagram data structure checking for other vertices to be deleted i.e., (a) find a vertex to be deleted; (b) test each of that vertex's neighbouring vertices for deletion; (c) when all neighbouring vertices of deleted vertices have been checked, then all vertices to be deleted must have been identified. The effectiveness of this approach depends on the efficiency with which the first vertex to be deleted can be found. If the list of points is ordered in a systematic manner, then a reasonable starting vertex is the last vertex in the structure. This procedure can lead to a negligible workload for step 3.

The specification of four initial points which define the convex hull within which all points lie is not, in practice, a restriction. These points can be chosen to ensure a sufficiently large hull so that no restrictions on the grid generators will occur.

It is apparent from the definition of a Voronoi polygon that problems can arise in the triangulation procedure when certain degeneracies occur in the data. Common degeneracies in two dimensions arise when

(1) two points are coincident,

(2) three points of a potential triangle lie on a straight line,

(3) four or more points are cyclic.

The first degeneracy is easily avoided by requiring that each new point be some small distance from its nearest neighbours before it is admitted into step 2 . The second degeneracy implies that the circumcentre of the three forming points is at infinity. This is readily detected and in such a case the new point which has caused a degeneracy can be rejected or moved a small distance and treated as a new point.

The third degeneracy is of a more subtle nature than the preceding two. In this case, two vertices are coincident and give rise to a point at which more than three Voronoi polygons meet. In such a situation, the resulting triangulation is not unique, but this in itself is not a problem. The triangulation procedure is still valid for such cases. However, this degeneracy may manifest itself at a later stage of the triangulation when a point is introduced which results in the deletion of one of the vertices of the Voronoi diagram but not the other. An inconsistency thereby arises in the vertex structure which can result in the breakdown of the non-overlapping triangulation procedure. This problem occurs because of round-off error within the computer. Baker (1987) has investigated this problem.

The motivation for the unstructured approach and the Delaunay triangulation was to provide a flexible method of producing meshes around complex shapes. In figure 33 a mesh is shown around a two-element high-lift aerofoil system. In this case two sets of points have been generated from a Von Karman-Trefftz transformation around the two components and the triangulation produced on the total points. A smoothing has been applied to the resulting point distribution. It is clear from figure 33 that high quality meshes can be produced from this approach.

In principle, this approach can be applied to $n$-multiply connected regions. A further example is shown in figure 34 for a 4 -element high-lift configuration. In this case 4-component meshes have been used with the mesh around the main element used 

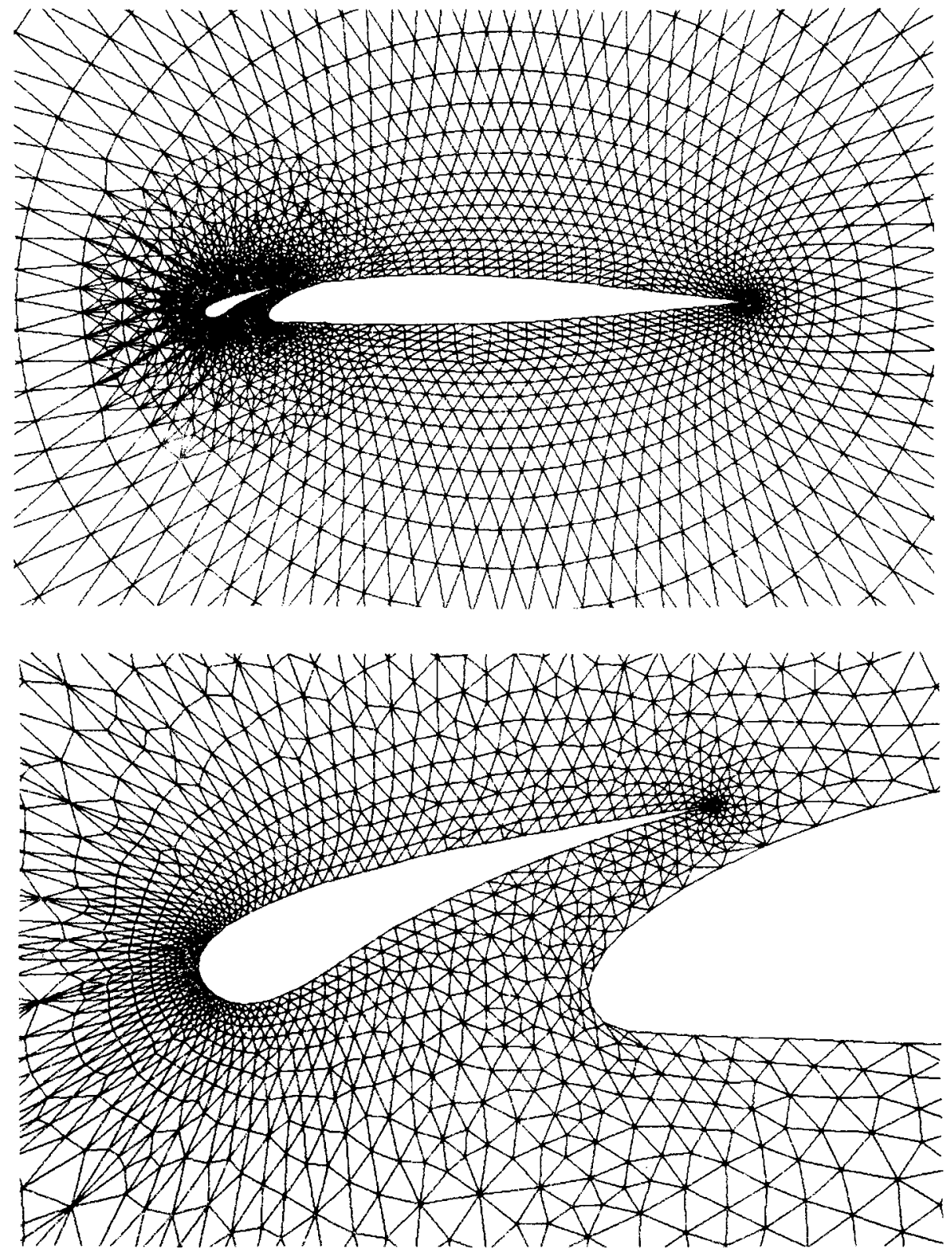

Figure 33. Triangulation around a two-element high-lift configuration.

to provide the points to the farfield boundary. Flowfield simulation can be performed on such meshes (Jameson \& Mavriplis 1985; James et al 1986: Mavriplis 1988) and the pressures on the 4-elements derived from the solution of the Euler equations are shown in figure 35 .

An example, taken from Baker (1987), of an application in 3 dimensions is given in figure 36. The transformation technique of Baker (1986), described in $\$ 2$, has been used to define suitable sets of mesh points around the components of Boeing 747 aircraft (Jameson \& Baker 1986, 1987; Baker 1987, 1988). The mesh points have then 


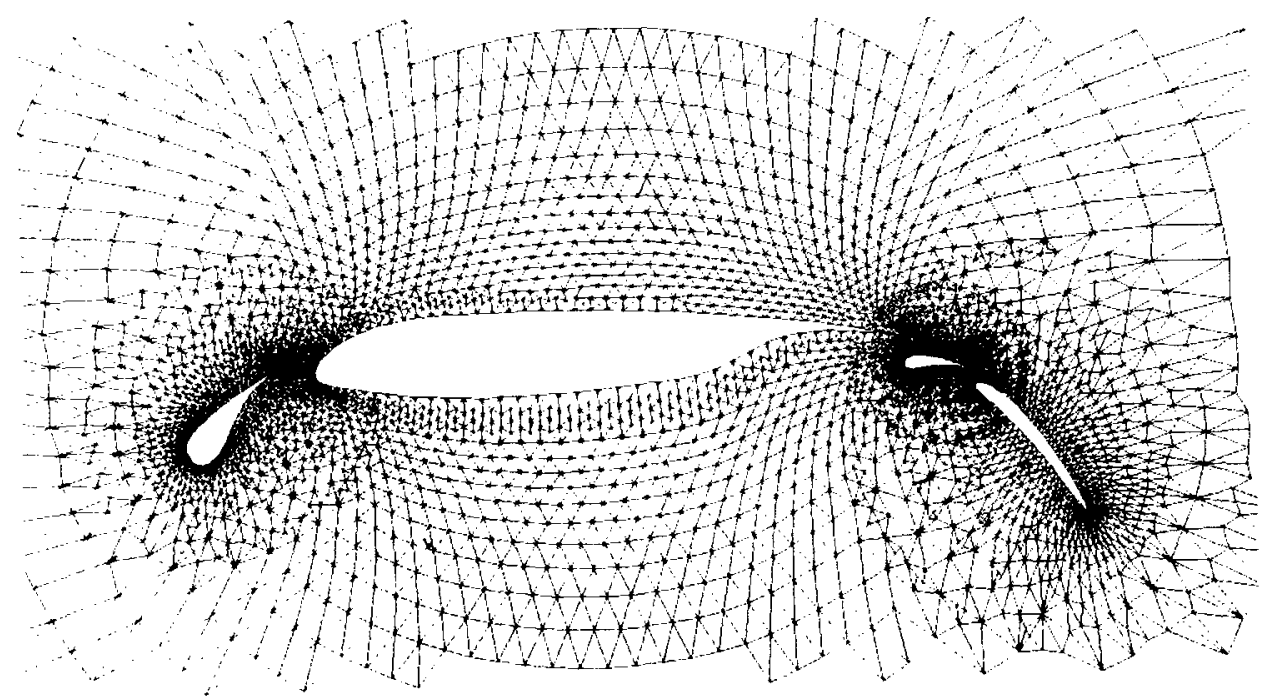

Figure 34. Unstructured mesh for- a high-lift configuration.

been connected using the Delaunay algorithm to produce a mesh of tetrahedral elements. In this simulation the flow algorithm developed by Jameson (Jameson et al 1986; Jameson \& Baker 1987) has been used to model the transonic flow over the configuration. It is perhaps worth commenting at this stage that unstructured grids in 3 dimensions do not lend themselves to visual presentation. This difficulty highlights the need for good quantitative measures of grid quality.

The Delaunay triangulation technique is now proving to be a popular method of providing 2- and 3-dimensional unstructured grids. Holmes \& Lamson (1986) have successfuliy applied the technique within an adaptive strategy for providing meshes
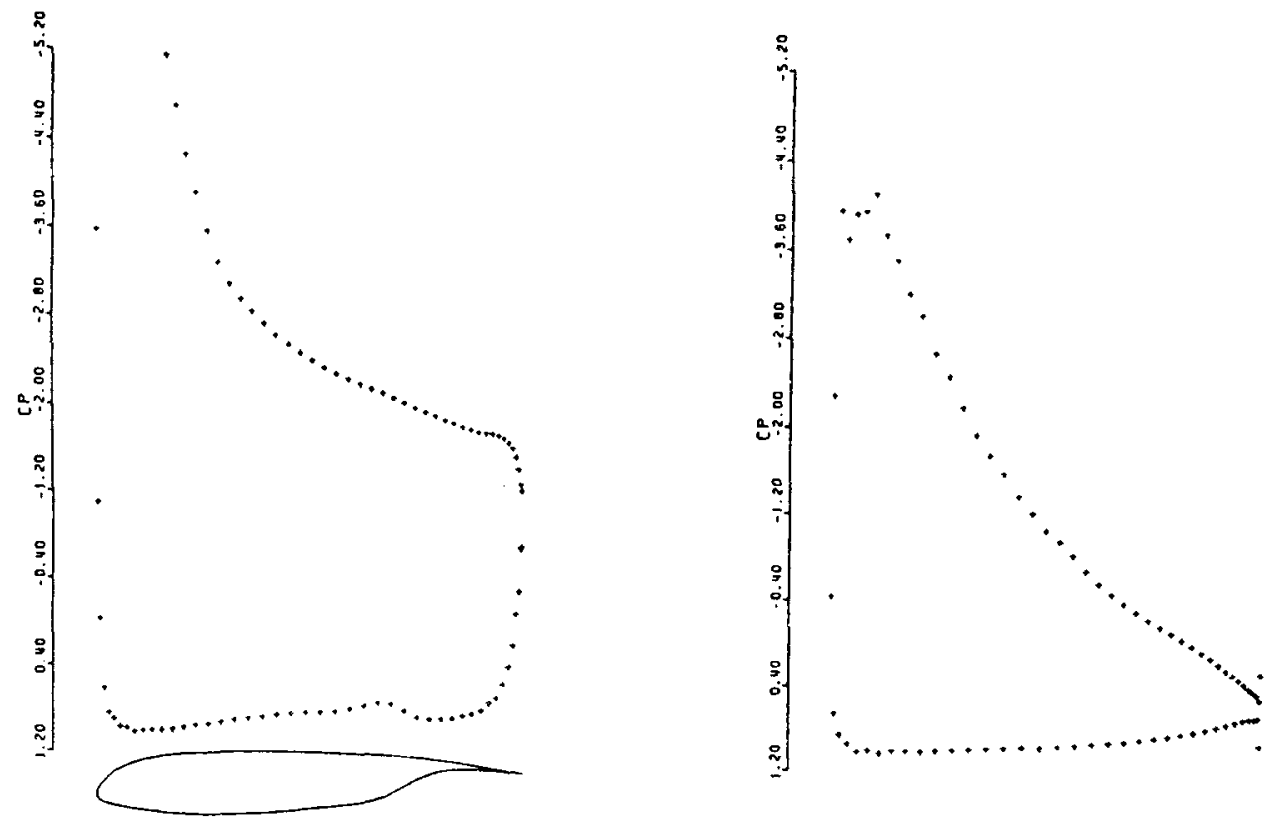

Figure 35. (Continued) 

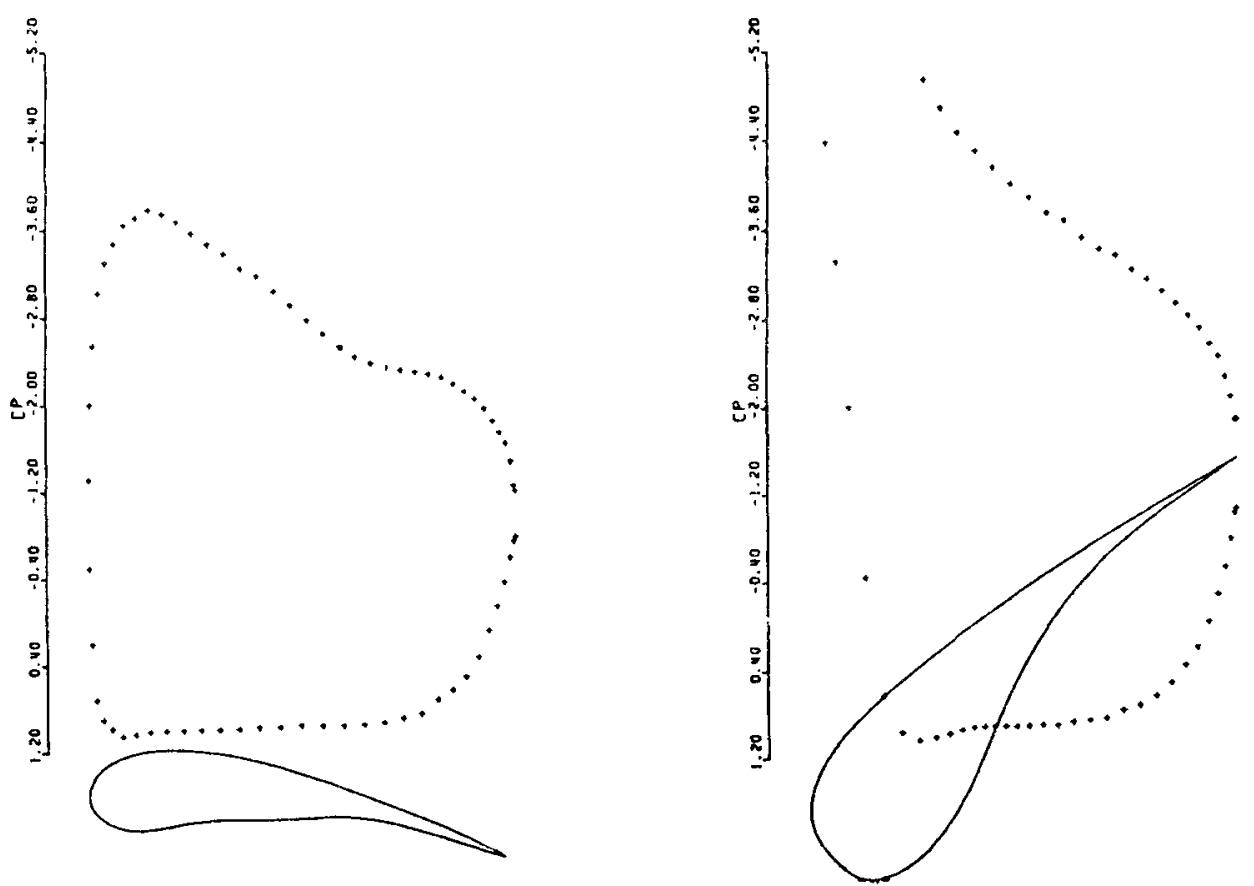

Figure 35. Pressure distribution on the surfaces of the configuration of figure 34 .

for turbo-machinery, whilst Mavriplis (1988) has used the method for accurate and efficient calculations for high-lift configurations. Further uses and details of different implementations of the idea can be found in other referenced works (Cavendish et al 1985).

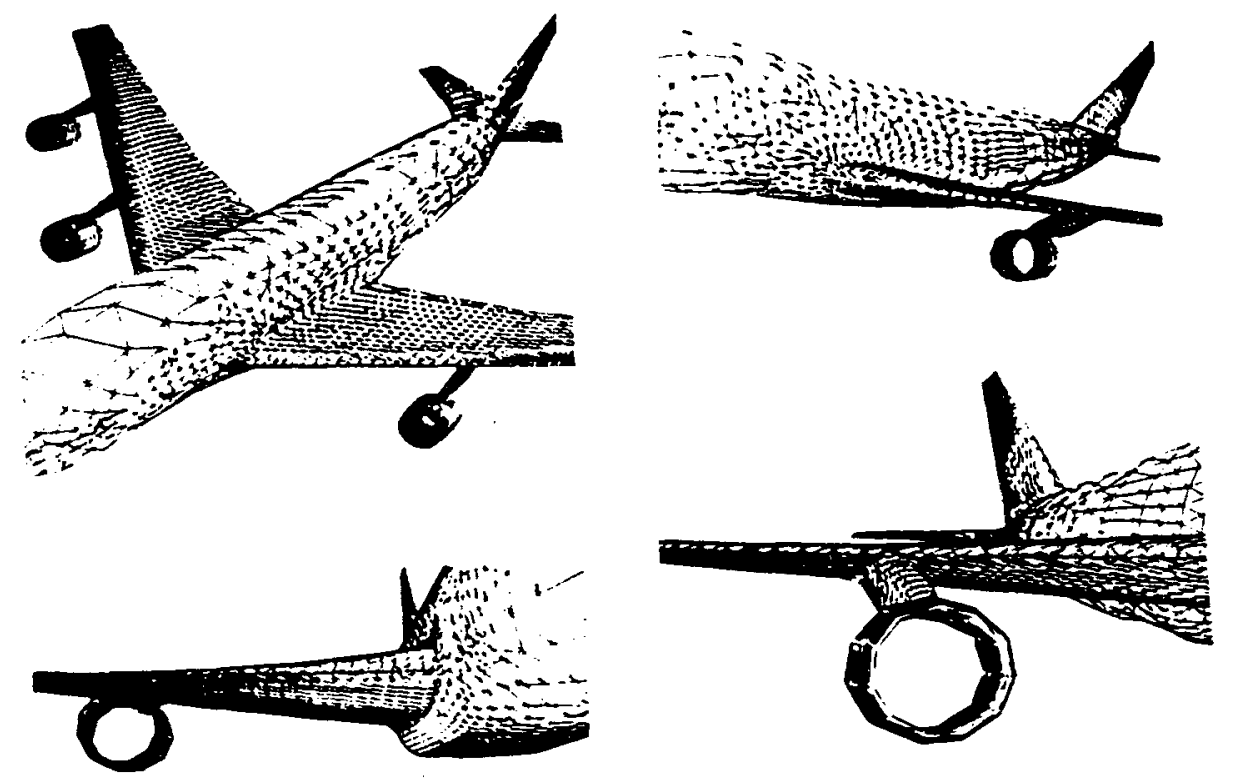

Figure 36. Surface mesh for a Boeing 747 (from Baker 1987). 


\section{Hybrid structured-unstructured meshes}

\subsection{Introduction}

In the preceding sections two contrasting techniques have been described which can generate meshes around complex shapes. It is easy to ask, but difficult to answer, which is the better approach? Undoubtedly the answer lies in how accurate the subsequent flow computations are on a given configuration on the different meshes. In time, detailed analysis of this type will emerge. However, the advanced state of development of these and other approaches together with a general acceptance that there are viable alternative methods based on either structured or unstructured methods has stimulated a general discussion on the apparent advantages of one methodology over the other. By general consent, the advantages of the structured approach are:

(1) the flexibility of implementation of all classes of flow algorithm,

(2) efficient utilisation of vector architecture of computers,

(3) efficient in CPU time and computer memory, and

(4) good environment for the multigrid technique for algorithm convergence.

The relative disadvantages include:

(1) lack of total flexibility for very complex geometrical regions, and

(2) not generally amenable to mesh point enrichment.

The unstructured method is:

(1) flexible for very complicated geometrical shapes,

(2) is a natural environment for mesh/flow adaptivity, and

(3) most suitable for transient computations in which adaptivity to moving flow features is essential.

Some disadvantages include:

(1) not necessarily amenable to all classes of flow algorithms (e.g. alternating direction implicit) and to the implementation of multigrid, and

(2) relatively inefficient in computer memory.

It is interesting to note that the real advantages of one of the approaches are the disadvantages of the other. This observation is at the heart of some recent work which attempts to marry these two approaches. Here we describe some initial work into this unification of structured-unstructured meshes. Three major applications of the hybrid approach are presented: (i) to encompass mesh enrichment/flow adaptivity, (ii) to improve mesh quality, and (iii) to utilise the flexibility of unstructured meshes for complex shapes.

\subsection{Mesh quality improvement}

This first application of hybrid meshes is based on the premise that a structured mesh has been generated but is found to be deficient in quality in some region or regions of the domain. In a conventional sense such a mesh would be interrogated and then user judgement would decide what remedial action is required. Usually, this action would take the form of a modification to the mesh control parameters, or, in some 


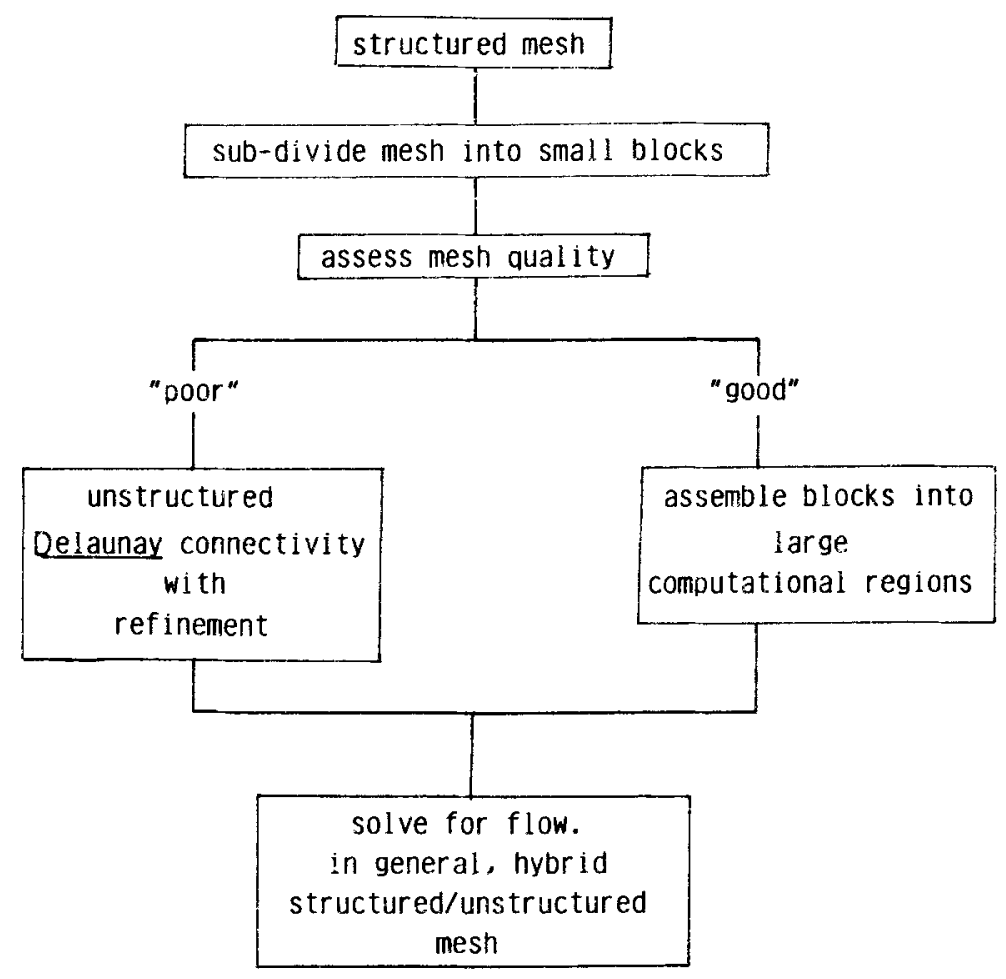

Figure 37. Strategy for mesh quality improvement.

cases, manual changes to the mesh could be made with the use of an interactive graphics facility. Here it is proposed that the mesh correction could be made by the use of localised unstructured meshes.

The general procedure is described in figure 37. A structured mesh is sub-divided into micro-blocks. Within each micro-block the mesh is analysed for quality. This includes mesh folding, mesh point distribution, mesh aspect ratio and mesh skewness. If the mesh within the micro-block is found to be of poor quality it is flagged for refinement and triangulation. If the quality is adequate the cells within the micro-block remain quadrilateral. All micro-blocks which remain quadrilateral are then ressembled to form a macro-block. Clearly, a flow algorithm working on the micro-blocks would be computationally inefficient as vector loops would be small. The final mesh consists of triangles and quadrilaterals in the form of a set of unstructured data and a set of large macro-blocks within which the mesh is structured.

An example of the application of this strategy is shown in figure 38 . The initial $\mathrm{H}$-mesh around the aerofoil contains poor quality shaped cells at the leading and trailing edges. These deficiencies are sufficient to have an adverse effect on the flowfield. However, following the procedure outlined and a suitable technique for mesh refinement the flow results obtained on the hybrid grid show a marked improvement.

The local refinement used here was based on an iterative procedure in which points are added such that the triangles, obtained by a Delaunay triangulation of the data points in the vicinity of the aerofoil, have an aspect ratio close to unity (Weatherill 1988). Other refinement techniques have been investigated.

This result is perhaps not so surprising. Obviously, the quality of any poor mesh 


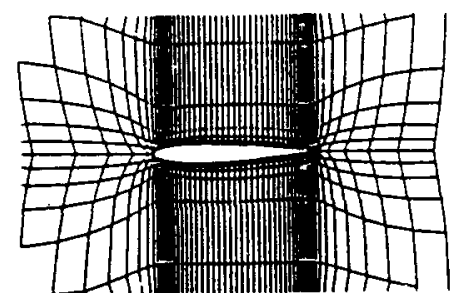

a)

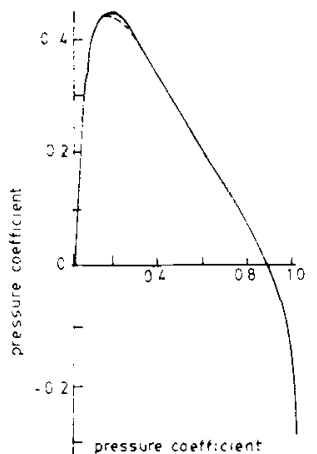

b)
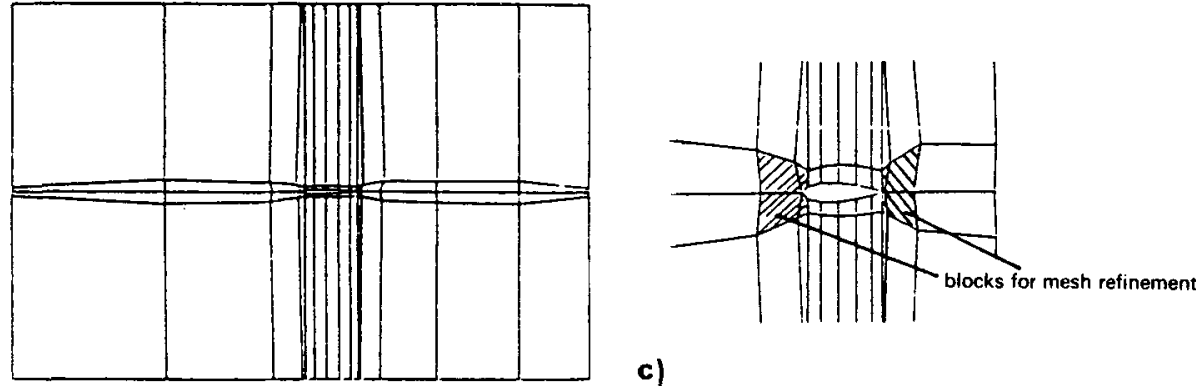

c)

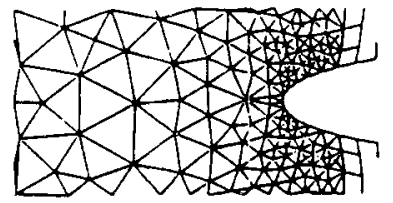

d)
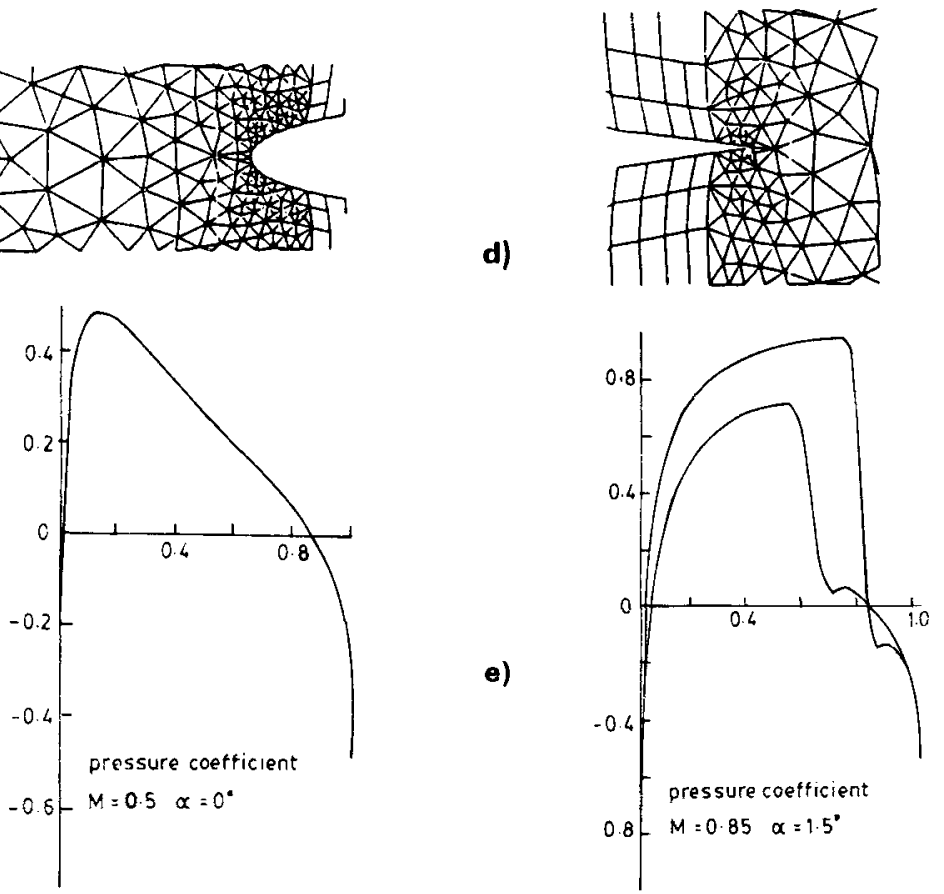

Figure 38. Example of mesh quality improvement.

can be improved if new points are added and a triangulation performed. However, the approach can prove useful when other attempts to improve the quality of a mesh have failed. The general applicability of the method relies upon the automation of mesh quality assessment. Obviously, user intervention could be relied upon to specify 
which, if any, of the blocks need to be refined but this is contrary to the philosophy of automatic mesh generation. A simple test can be performed to assess if a cell has a negative area. In such cases the block which contains the cell can be refined and the points triangulated. However, the criteria for the test of mesh density, mesh skewness and mesh aspect ratio are less easy to define and further work continues in this area.

\subsection{Meshes for complex geometries}

7.3a General approach: The approach of Jameson et al (1986) discussed in $\S 6$ was to generate grids around each component of a configuration and then connect all the points using a Delaunay triangulation. Steger et al (1983) followed a strategy of component meshes but allowed all meshes to overlap one another. Through this overlap, flow information was transferred using interpolation from one mesh to another.

Here, it is proposed that a global mesh is generated and to accommodate other components holes are cut and the component meshes inserted. The component meshes remain structured wherever geometrically possible and are connected to the global mesh by constructing 'ribbons' of triangles. This technique is not only a valid mesh generation approach but also can be used to further enhance a structured mesh which has been generated from some other technique, e.g. multiblock. The general procedure is shown in figure 39.

7.3b A family of meshes for multiply connected domains: The procedure outlined in $\$ 7.3 \mathrm{a}$ and minor modifications to it can be used to generate a family of meshes. Here, these mesh types will be illustrated on a two-element aerofoil configuration.
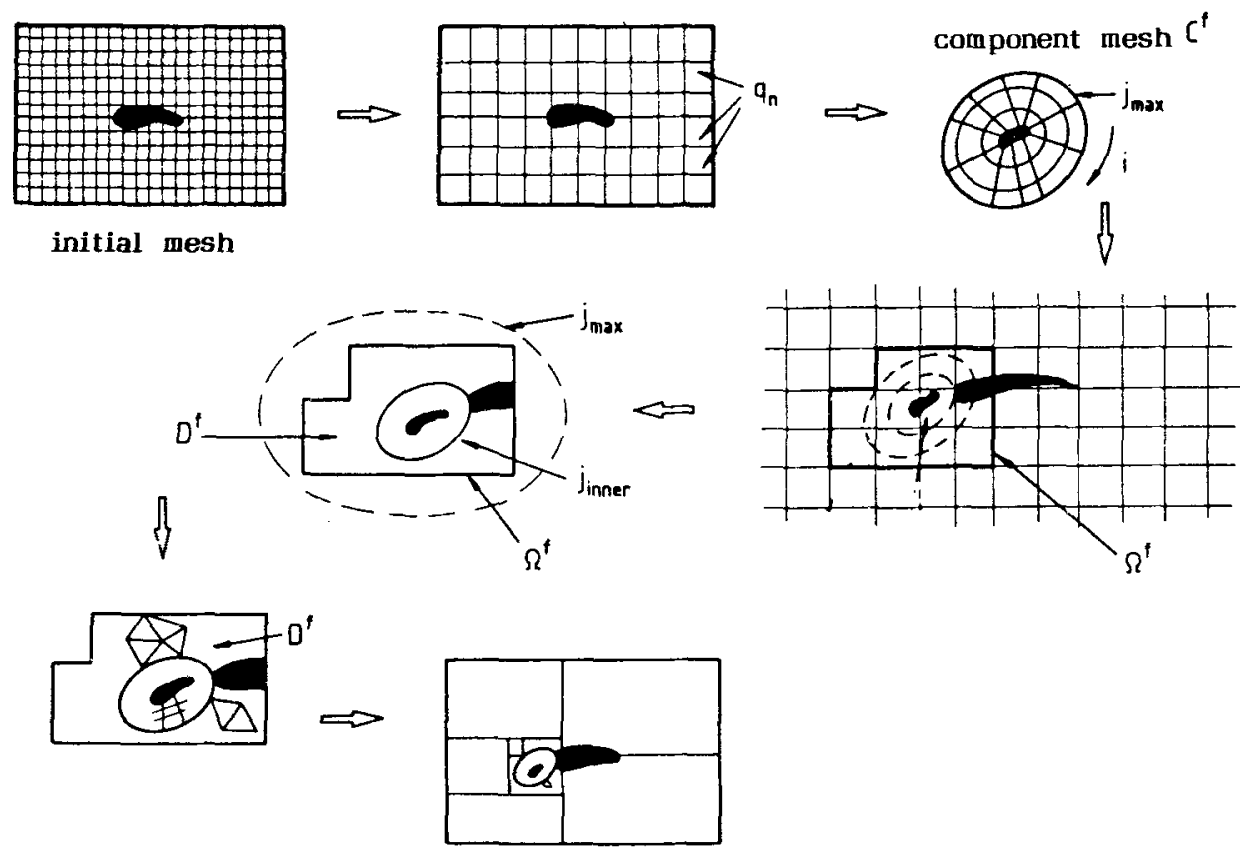

Figure 39. Strategy for complex geometries. 


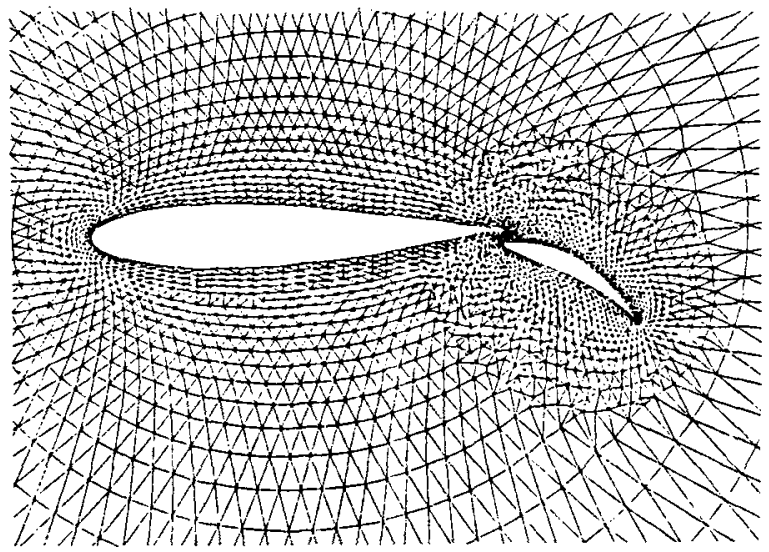

Figure 40. Delaunay mesh - completely unstructured.

Firstly, referring back to the approach described in $\$ 7$, meshes around each component are generated and then all the points are triangulated. For the configuration of interest such a mesh is shown in figure 40 . In this mesh, away from the region in which the points from the flap interfere with the points from the main aerofoil, the mesh can be seen to possess a lot of structure within the triangulation. Figure 41 shows a mesh in which the region in the block $D^{f}$ is triangulated although much of the global mesh has retained its original quadrilateral structure. To further extend the range of meshes based on these concepts, figure 42 now shows another mesh in which a structured mesh has been retained close to the flap. The extent of the structured mesh is restricted by the proximity of the main aerofoil.

The unstructured mesh, however, away from the gap region retains much of its original structure and therefore it would be appropriate to utilise as much of this structure as possible. Figure 43 shows a case in which the structured mesh within the cut-out region of the main mesh is allowed to extend as far as possible without crossing the boundary of the global mesh. This empty 'ribbon' region is then triangulated using, in this case, a Delaunay algorithm, but alternative triangulation schemes could be used. In this latter case quadrilateral cells are retained in as much of the flow domain as possible.

Figures 40 to 43 have been presented for a typical medium mesh for an inviscid flow simulation. However, the value of inviscid flow simulation for multi-component

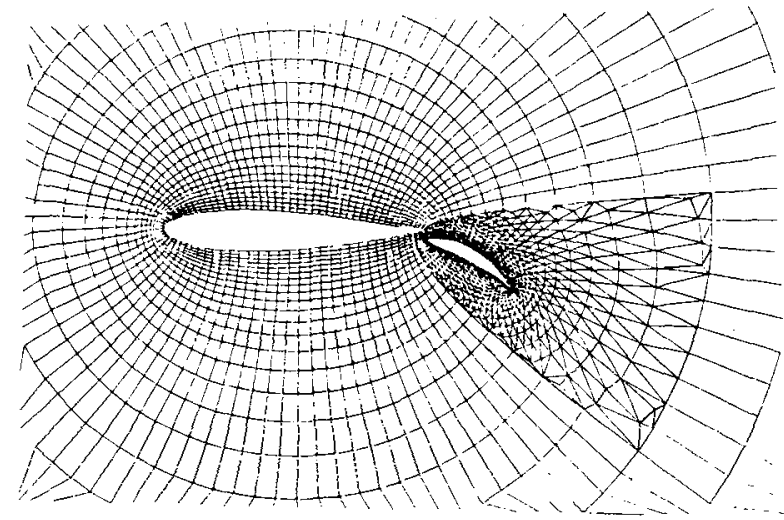

Figure 41. Hybrid mesh-unstructured around the flap. 


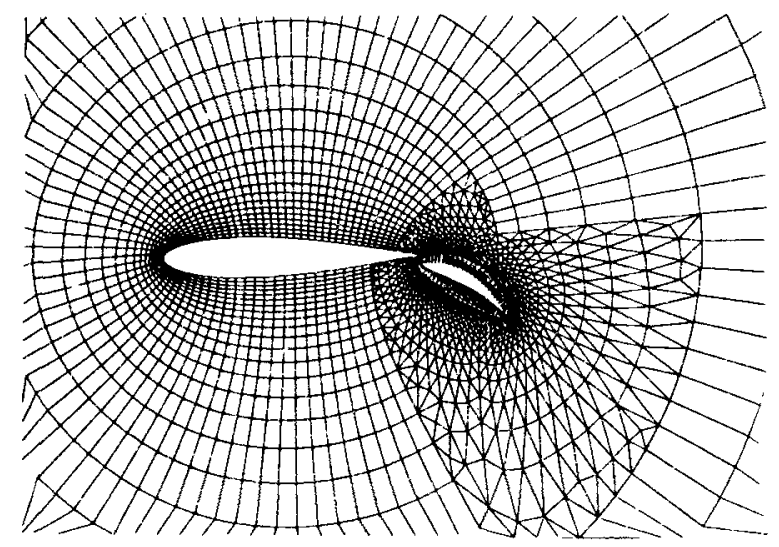

Figure 42. Hybrid mesh.

configurations is limited and the simulation of the viscous flow is of more importance. Hence, in figure 44, a mesh suitable for viscous flow simulation is shown.

In this case stretched meshes with a high density of points close to the main aerofoil surface have been generated using a conformal mapping technique. For both aerofoils a near-wall structured mesh has been preserved. These near-wall structured meshes are used to provide a suitable mesh and data structure for implementation of modifications to the dissipation operator and turbulence models (Weatherill et al 1987) or to alleviate time step limitations in the highly stretched cells near the wall by the use of an implicit flow algorithm in this region (Hassan et al 1989).

Flow algorithms to simulate inviscid and viscous flows on such hybrid meshes have been developed (Weatherill 1988). Pressure distributions for the flap configuration are shown in figure 45 .

\subsection{Mesh adaptivity}

Many different approaches to flow adaptivity exist both for structured and unstructured meshes. It was noted that the technique used in the former often resulted in an unstructured data system. In keeping with the theme of this chapter the strategy for the implementation of adaptivity within a hybrid grid is shown in figure 46.

To illustrate the strategy for mesh adaption, assume that a mesh around an NACA0012 aerofoil is given and that no improvements in its quality are required. The

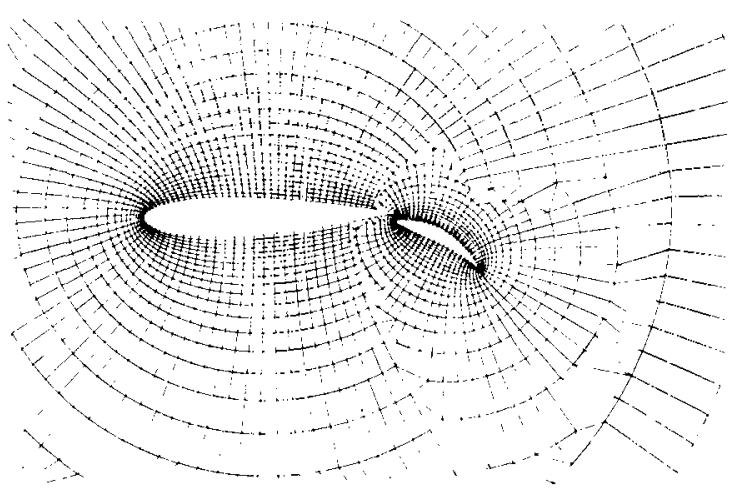

Figure 43. Hyrbid mesh which maximises the use of structured quadrilaterals. 


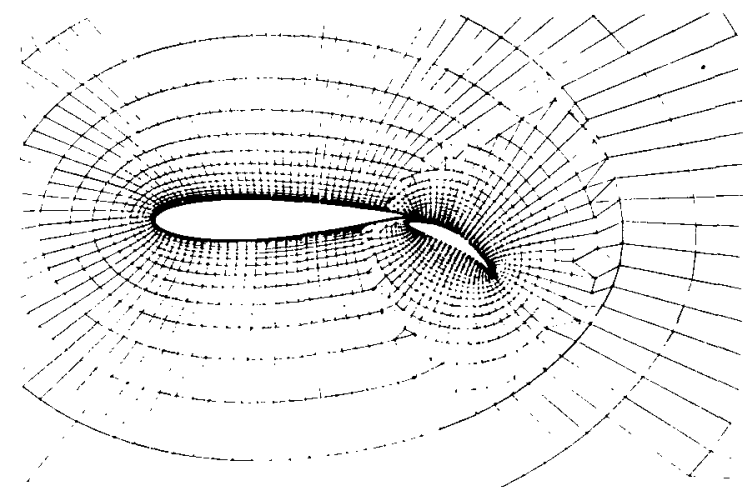

Figure 44. Hybrid mesh for viscous flow simulations.

strategy suggests that the Euler algorithm is used on the quadrilateral mesh to establish the flow features. This achieved, the flow domain is then subdivided into small segments. The algorithm developed to perform this decomposition required as input the dimensions of the smallest segment. The flowfield is examined to determine the cells in which large flow gradients are present. All blocks for which more than half the number of celis have large gradients are then flagged for triangulation. After this analysis the assembly of all blocks with quadrilateral cells is then performed. For an initial polar mesh of $80 \times 25$ grid points and with a subdivision size of $4 \times 4$, 104 blocks are produced. Mesh adaptivity based upon the gradient of pressure for the flowfield of $\mathbf{M}=0.85$, incidence $=0$, results in 4 blocks for mesh refinement and 3 structured computational blocks with dimensions $(51 \times 24),(24 \times 10)$ and $(31 \times 15)$ after assembly. The hybrid mesh and the pressure contours obtained from the flow simulation are shown in figures 47 and 48 respectively.

\subsection{Efficiency of the hybrid approach}

Flow results presented in figure 48 for the hybrid process were obtained a factor of 2.5 times faster than the calculation performed on the mesh when the unstructured
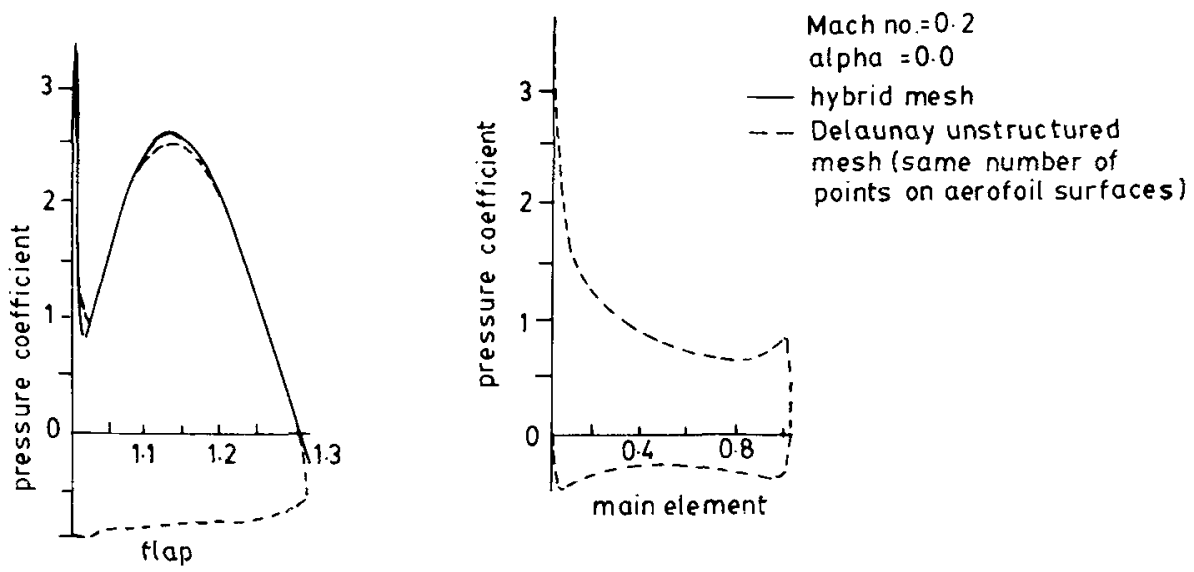

Figure 45. Pressure distributions on the flap configuration. 


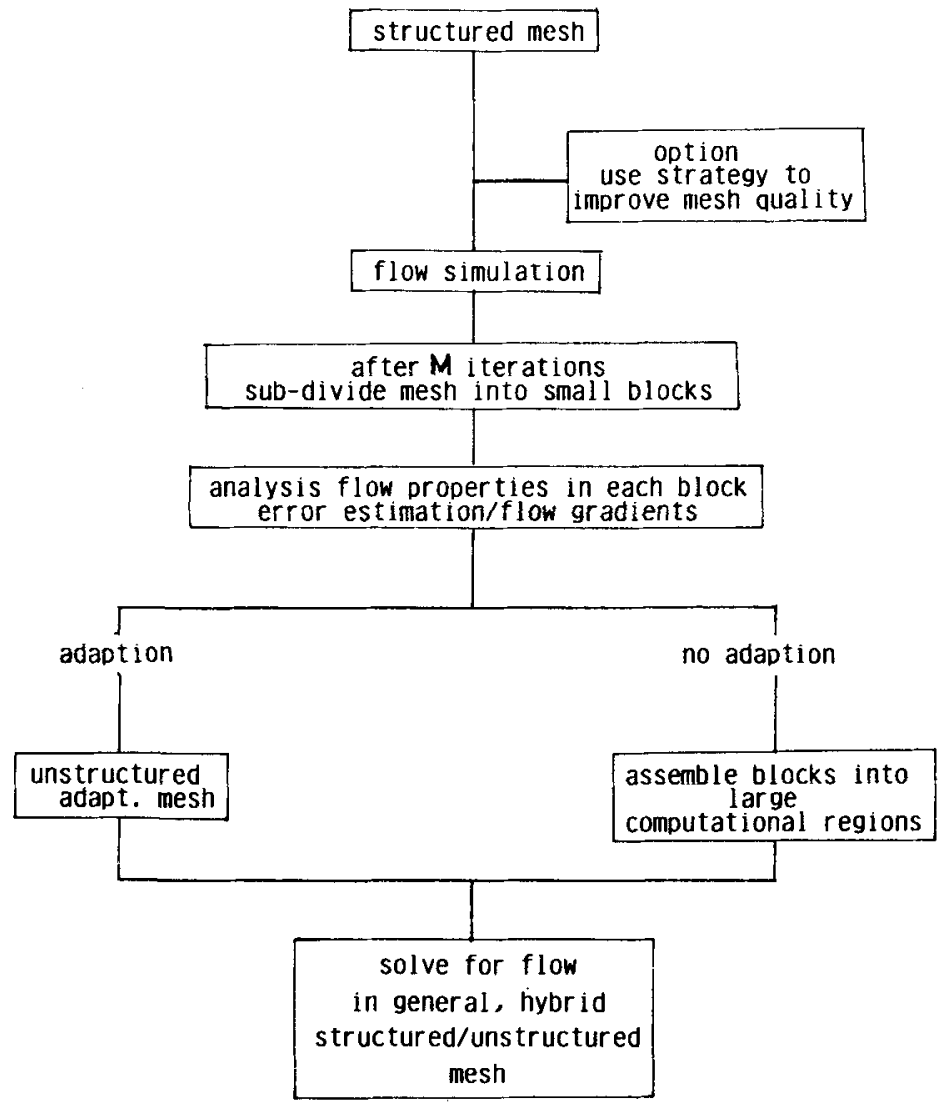

Figure 46. Strategy for mesh adaptivity.

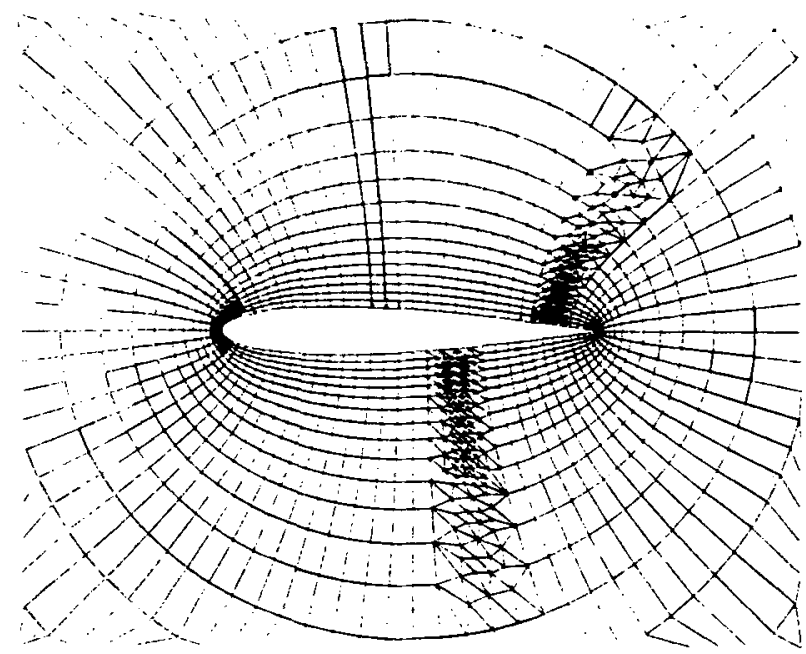

Figure 47. Mesh adaption on a hybrid mesh. 

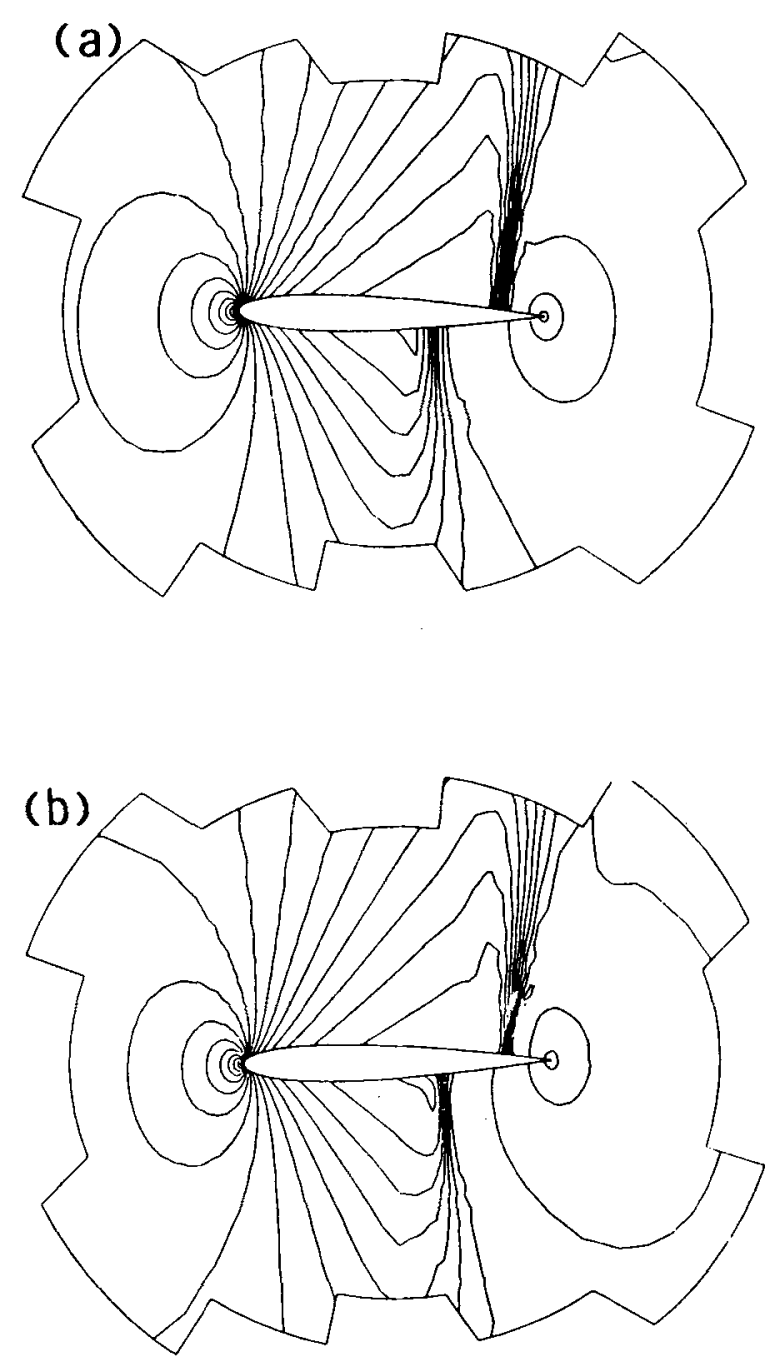

Figure 48. Mesh adaptivity: flow contours of pressure before (a) and after (b) refinement. Mach no. $0 \cdot 8$; incidence $=1.5$.

unvectorised triangle data structure was used throughout the domain. This factor has been achieved by minimising the computations required by utilising quadrilaterals in the majority of the flowfield and capitalising on the relatively long vector lengths achievable in the three structured regions. Clearly, the vectorisation factor is heavily dependent on how the small segments are reassembled and figure 49 shows a plot of efficiency versus number of assembled structured regions. More rectangles, for a given number of mesh points, imply small vector lengths and with this lower computational speed.

\section{Discussion}

This paper, which closely follows the presentation given at the CAARC Specialists' Meeting in 1988, provides a brief overview of mesh generation techniques for computational fluid dynamics. Unfortunately, due to space limitations some popular 


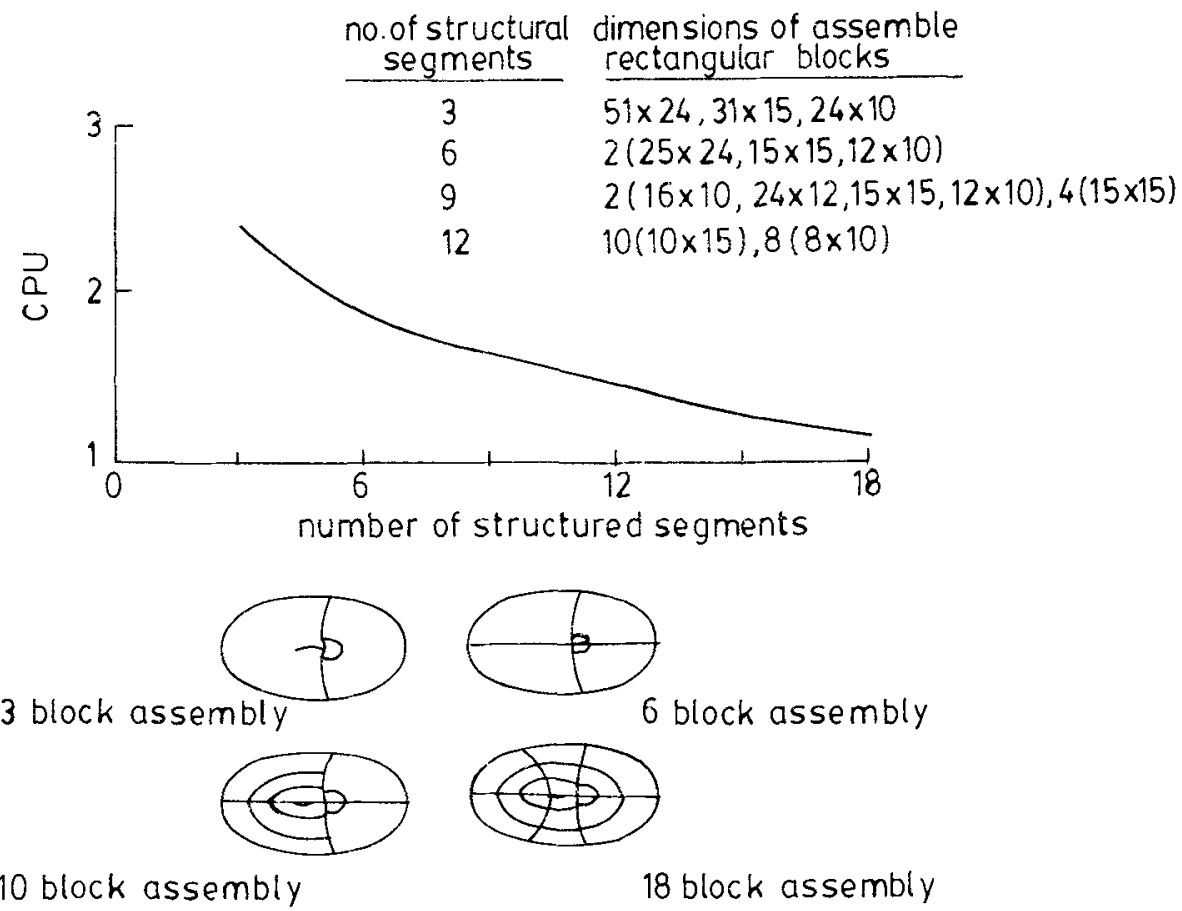

Figure 49. Efficiency of the hybrid approach.

mesh generation methods have not been given the attention that they deserve. Furthermore, the important area of mesh adaptivity has been largely ignored.

The author is grateful to the Royal Aerospace Establishment, Farnborough, and the Aircraft Research Association for their support of some of the work described. Particular acknowledgement is given to Mr C R Forsey, Dr K E Rose and Mr J A Shaw of ARA for their major contributions in the development of the multiblock method.

\section{References}

Albone C M 1988 An approach to geometric and flow complexity using feature-associated mesh embedding (FAME): Strategy and ñrst results. Numerical methods for fluid dynamics (3)(eds) K W Morton, M J Baines (Oxford: University Press)

Arina R 1986 Orthogonal girds with adaptive control, Numerical grid generation in CFD (eds) J Hauser, C Taylor (Swansea: Pineridge)

Baker T J 1986 Mesh generation by a sequence of transformations. J. Appl. Numer. Math. 2: 515-528

Baker T J 1987 Three-dimensional mesh generation by triangulation of arbitrary point sets. Proceedings of the AIAA 8 th CFD Conference (Swansea: Pineridge)

Baker T J 1988 In Numerical grid generation in CFD (eds) E Sengupta, J Hauser, P Eiseman, J F Thompson (Swansea: Pineridge)

Baxendale A J 1988 Application of CFD to military aircraft at supersonic speeds. Proceedings of the Royal Aeronautical Society Symposium on Aerodynamics Design for Supersonic Flight (London: R. Aeronant. Soc.) 
Benek J A, Buning P G, Steger J L 1985 A 3-dimensional chimera grid embedding technique. Proceedings of the 7th AIAA CFD Conference, pp 322-331

Boerstoel J W 1988 Numerical grid generation in 3-dimensional Euler flow simulation. In Numerical methods in fluid dynamics (3) (eds) K W Morton, M J Baines (Oxford: University Press) p. 187

Bowyer A 1981 Computing Dirichlet tessellations. Comput. J. 24: 162-166

Cavendish J C, Field D A, Frey W H 1985 An approach to automatic three-dimensional finite element mesh generation. Int. J. Numer. Methods Eng. 21: 329-347

Coleman R M 1982 Generation of boundary-fitted coordinate systems using segmented computational regions. Numerical grid generation (ed.) J F Thompson (Amsterdam: North Holland)

Dirichlet G L 1850 Uber die Reduction der positivien Quadratischen formen mit drei Undestimmten ganzen Zahlen. Z. Reine Angew. Math. 40: 209-227

Edwards T A 1985 Non-iterative 3-dimensional grid generation using parabolic partial differential equations, 23rd Aerospaces Sciences Meeting, AIAA paper-85-0485

Eriksson L E 1981 Transfinite interpolation, VKI lecture series in CFD (Brussels: Von Karman Inst.)

Eriksson L E 1982 Generation of boundary-conforming grids around wing-body configurations using transfinite interpolation. AIAA J. 20: 1313-1320

Faux I D, Pratt M J 1979 Computational geometry for design and manufacture (London: Ellis-Horwood)

Floryan J M 1983 Coordinate generation method for flows through periodic channels, Jnt. Conf. Numerical methods in laminar and turbulent flow (3) (Swansea: Pineridge) pp. 1194-1203

Forsey C R, Billing C M 1988 Some experiences with grid generation on curved surfaces using variational and optimisation techniques. In Numerical methods for fluid dynamics (3) (eds) K W Morton, M J Baines (Oxford: University Press)

Fortune S 1985 A sweepline algorithm for Voronoi diagrams, AT + T Bell Laboratory report, Murray Hill, NJ

Fulker J L, Ashill P R 1988 A theoretical and experimental evaluation of a numerical method for calculating supersonic flows over wing-body configurations, AGARD paper, Lisbon

George A J 1971 Computer implementation of the finite element method, Stanford University, Department of Computer Science, Stan-CS071-208

Gordon W J, Hall C A 1973 Construction of curvilinear coordinate systems and application to mesh generation. Int. J. Numer. Methods Eng. 7: 461-477

Green P J, Sibson R 1978 Computing Dirichlet tessellations in the plane. Comput. J. 24: 168-173

Halsey N D 1979 Potential flow analysis of multielement airfoils using conformal mappings. AIAA J. 17: $1281-1288$

Halsey N D 1986 Conformal mapping as an aid in grid generation for complex 3-dimensional configurations, AIAA paper 1986

Hassan O, Morgan K, Peraire J 1989 An adaptive implicit/explicit finite element scheme for compressible viscous high speed flows, 27th Aerospace Sciences Meeting (Reno), AIAA paper 89-0363

Hauser J, Taylor C (eds) 1986 Numerical grid generation in CFD (Swansea: Pineridge)

Hauser J, Sengupta E, Eiseman P, Thompson J F (eds) 1988 Numerical grid generation in CFD (Swansea: Pineridge)

Holmes D G, Lamson S H 1986 Adaptive triangular meshes for compressible flow simulations. Numerical grid generation in CFD (eds) J Hauser, C Taylor (Swansea: Pineridge)

Ives D C 1975 A modern look at conformal mapping, including doubly connected regions, AIAA paper 75-842

Jameson A, Baker T J 1987 Improvements to the aircraft Euler method, 25th Aerospace Science Meeting (Reno), AIAA paper-87-0452

Jameson A, Baker T J, Weatherill N P 1986 Calculation of inviscid transonic flow over a complete aircraft. 24th AIAA Aerospaces Sciences Meeting (Reno), AIAA paper 86-0103

Jameson A, Mavriplis D 1985 Finite volume solution of the 2-dimensional Euler equations on a regular triangular mesh, 23rd AIAA Aerospaces Sciences Meeting (Reno), AIAA paper 85-0435

Joukowski N 1910 Ueber die Konturen der drachen Flieger. Z. Flugtech. Motorluftsch. 1: 281

Kober H 1952 Dictionary of conformal representation (New York: Dover)

Mavriplis D J 1988 Accurate multigrid solution of the Euler equations on unstructured and adaptive meshes, First National Fluid Dynamics Congress (Cincinnati, OH), AIAA paper 88-3707

Miki K, Tagagi T 1984 A domain decomposition and overlapping method for the generation of three-dimensional boundary-fitted coordinate systems. J. Comput. Phys. 53: 319-330

Milne-Thompson L M 1968 Theoretical hydrodynamics 5th edn (London: Macmillan)

Moretti G 1980 Grid generation using classical techniques, NASA Conference Publication 2166, Numerical Grid Generation Techniques

Nakahashi K, Obayashi S 1987 FDM-FEM zonal approach for viscous flow computations over multiple bodies, 25th Aerospaces Sciences Meeting (Reno), AIAA paper 87-0604 
Nakamura S 1982 Marching grid equations using parabolic partial differential equations. Numerical grid generation (ed.) J F Thompson (Amsterdam: North Holland)

Peraire $\mathrm{J} 1986$ A finite element method for convection dominated flows, Ph D thesis, University of Wales

Peraire J, Vahdati M, Morgan K, Zienkiewicz O C 1987 Adaptive remeshing for compressible now computations. J. Comput. Phys. 72: 449--466

Roberts A 1982 Automatic topology generation and generalised B-spline mapping. Numerical grid generation (ed.) J F Thompson (Amsterdam: North Holland)

Schwarz W 1986 Elliptic grid generation system for 3-dimensional configurations using Poisson equation. Numerical grid generation in CFD (eds) J Hauser, C Taylor (Swansea: Pineridge)

Shaw J A, Forsey C R, Weatherill N P, Rose K E 1986 A block structured mesh generation technique for aerodynamic geometrics. Numerical grid generation in CFD (eds) J Hauser, C Taylor (Swanseą: Pineridge)

Shaw J A, Georgala J M, Weatherill N P 1988 The construction of component-adaptive grids for aerodynamic geonetrics. Proc. of Int. Conf. on Numerical Grid Generation in CFD (eds) E Sengupta, J Hauser, P Eiseman, I F Thompson (Swansea: Pineridge)

Sheppard M S 1988 Approaches to the automatic generation and control of finite element meshes. Appl. Mech. Rev. 41:

Sneddon I N 1957 Elements of partial differential equations (London: McGraw-Hill)

Sorenson R L, Steger J L 1979 Automatic mesh point clustering near a boundary in grid generation with elliptic partial differential equations. J. Comput. Phys. 33: 405

Steger J L, Dougherty F C, Benek J A 1983 A chimera grid scheme. Advances in grid generation (eds) N K Ghia, U Ghia

Steger J L, Sorenson R L 1980 Use of hyperbolic partial differential equations to generate body-fitted coordinates. Numerical grid generation (ed.) R E Smith, NASA Conference Publication, CP-2166

Thacker W C 1980 A brief review of techniques for generating irregular computational grids. Int. J. Numer. Mehods Eng. 15: 1335-1341

Thames F C 1984 Grid generation using elliptic partial differential equations and multignid techniques, NASA Langley Report

Thomas P D, Middlecoff J F 1980 Direct control of the grid point distribution in meshes generated by elliptic equations. AIAA $J$. 18: 652-656

Thompson J F 1987 A general 3-dimensional elliptic grid generation system on composite block structure. Comput. Methods Appl. Mech. Eng. 64: 377-411

Thompson J F, Thames F C, Mastin C W 1974 Automatic numerical grid generation of body-fitted curvilinear coordinate system of field containing and number of arbitrary 2-dimensional bodies. $J$. Comput. Phys. 15: 299-319

Thompson J F, Warsi Z, Mastin C W 1985 Numerical grid generation: Foundations and applications (Amsterdam: North-Holland)

Trefethen $\mathrm{L} N$ (ed.) 1986 Numerical conformal mapping (Amsterdam: North Holland)

Vigneron Y, Lejal T 1984 Calculation of transonic flows around an aircraft configuration with motorized nacelles, ICAS Conference paper 84-2.10.2

Von Karman T, Trefftz E 1918 Potential Stromungen um gegebene Tragflachen-Querschnitte. Z. Fluid Mech.

Watabayashi G Y, Galt J A 1986 An optimised triangular mesh system from random points. Numerical grid generation in CFD (eds) J Hauser, C Taylor (Swansea: Pineridge)

Watson D F 1981 Computing the $n$-dimensional Delaunay tessellation with application to Voronoi polytopes. Comput. J. 24: 167-172

Weatherill N P 1985 The generation of unstructured grids using Dirichlet tessellation, Princeton University, MAE report No. 1725

Weatherill N P 1988a A method for generating irregular computational grids in multiply connected planar domains. Int. J. Numer. Methods Fluids 8: 181-197

Weatherill N P 1988b An investigation into the use of hybrid structured-unstructured meshes in computational aerodynamics, Part I and Part II. Institute for Numerical Methods in Engineering, University College of Swansea, Report No. CR/604/88 and CR/605/88

Weatherill N P, Forsey C R 1985 Grid generation and flow calculations for a aircraft geometries. J. Aircraft 22: 855-860

Weatherill N P, Johnston L J, Peace A J, Shaw J A 1987 Navier-Stokes calculations on unstructured grids, ARA report

Weatherill N P, Shaw J A 1988 Component adaptive grid generation for aircraft configurations AGARDOGRAPH (eds) H Yoshihara, J L Steger, J F Thompson (Paris: AGaRd)

Weatherill N P, Shaw I A, Forsey C R, Rose K E 1986 A discussion on a mesh generation technique applicable to complex geometries. $A G A R D$ Conference Proceedings No. 415 on application of $C F D$ in Aeronautics (Paris: AGARD) 